\title{
Frobenius' Idea Together with Integral Bifurcation Method for Investigating Exact Solutions to a Water Wave Model of the Generalized mKdV Equation
}

\author{
Weiguo Rui \\ College of Mathematics, Chongqing Normal University, Chongqing 401331, China \\ Correspondence should be addressed to Weiguo Rui; weiguorhhu@aliyun.com
}

Received 19 May 2014; Accepted 30 July 2014

Academic Editor: Salvatore Alfonzetti

Copyright (C) 2015 Weiguo Rui. This is an open access article distributed under the Creative Commons Attribution License, which permits unrestricted use, distribution, and reproduction in any medium, provided the original work is properly cited.

\begin{abstract}
By using Frobenius' idea together with integral bifurcation method, we study a third order nonlinear equation of generalization form of the modified KdV equation, which is an important water wave model. Some exact traveling wave solutions such as smooth solitary wave solutions, nonsmooth peakon solutions, kink and antikink wave solutions, periodic wave solutions of Jacobian elliptic function type, and rational function solution are obtained. And we show their profiles and discuss their dynamic properties aim at some typical solutions. Though the types of these solutions obtained in this work are not new and they are familiar types, they did not appear in any existing literatures because the equation $u_{t}+u_{x}+v u_{x x t}+\beta u_{x x x}+\alpha u u_{x}+(1 / 3) v \alpha\left(u u_{x x x}+2 u_{x} u_{x x}\right)+3 \mu \alpha^{2} u^{2} u_{x}+$ $\nu \mu \alpha^{2}\left(u^{2} u_{x x x}+u_{x}^{3}+4 u u_{x} u_{x x}\right)+v^{2} \mu \alpha^{2}\left(u_{x}^{2} u_{x x x}+2 u_{x} u_{x x}^{2}\right)=0$ is very complex. Particularly, compared with the cited references, all results obtained in this paper are new.
\end{abstract}

\section{Introduction}

It has come to light that many problems in nonlinear science associated with mechanical, structural, aeronautical, oceanic, electrical, and control systems can be summarized as solving nonlinear partial differential equations (PDEs) which arise from important models with mathematical and physical significances. Finding their exact solutions has extensive applications in many scientific fields such as hydrodynamics, condensed matter physics, solid-state physics, nonlinear optics, neurodynamics, crystal dislocation, model of meteorology, water wave model of oceanography, and fibre-optic communication. The research methods for solving nonlinear PDEs deal with the inverse scattering transformation $[1,2]$, the Darboux transformation [3-5], the Bäcklund transformation [5-8], the bilinear method and multilinear method $[9,10]$, the classical and nonclassical Lie group approaches [11, 12], the Clarkson-Kruskal direct method [13-15], the mixing exponential method [16], the geometrical method [17, $18]$, the truncated Painlevé expansion $[19,20]$, the function expansion method (including tanh expansion method [21, 22], sine-cosine expansion method [23, 24], exp-function method [25], and multiple exp-function method [26]), the bifurcation theory of planar dynamical system [27, 28], the F-expansion type method [29,30], $G^{\prime} / G$ method [31, 32], and the integral bifurcation method [33-36]. Among these available methods for solving nonlinear PDEs, some of them employed Frobenius' idea directly or indirectly. Frobenius' idea is aso called Frobenius' integrable decompositions [37]; it can reduce a partial differential equation (PDE) to an ordinary differential equation (ODE) under investigation for solution. Indeed, the F-expansion type methods indirectly employed Frobenius' idea; crucial points of this method are to choose integrable ODE to start investigation for solution. In fact, the tanh function method and $G^{\prime} / G$ method are special cases of such an idea or general Frobenius' idea. Direct Frobenius' idea was also used to establish the transformed rational function method [38] and to solve the KPP equation [39]. 
In this paper, we will employ Frobenius' idea together with integral bifurcation method to investigate exact traveling wave solutions of the following integrable generalization of the modified KdV equation:

$$
\begin{aligned}
u_{t}+ & u_{x}+v u_{x x t}+\beta u_{x x x}+\alpha u u_{x} \\
& +\frac{1}{3} v \alpha\left(u u_{x x x}+2 u_{x} u_{x x}\right)+3 \mu \alpha^{2} u^{2} u_{x} \\
& +\nu \mu \alpha^{2}\left(u^{2} u_{x x x}+u_{x}^{3}+4 u u_{x} u_{x x}\right) \\
& +v^{2} \mu \alpha^{2}\left(u_{x}^{2} u_{x x x}+2 u_{x} u_{x x}^{2}\right)=0
\end{aligned}
$$

where $\alpha, \beta, \mu$, and $\nu$ are constants and $0<\alpha<1$. The model (1) comes from the physical and asymptotic considerations via the methodology introduced by Fokas [40] in 1995; it can be regarded as a water wave model to describe the motion of water wave. It is worth to point out that the special case of (1),

$$
\begin{aligned}
u_{t} & +u_{x}+v u_{x x t}+\beta u_{x x x}+\alpha u u_{x} \\
& +\frac{1}{3} v \alpha\left(u u_{x x x}+2 u_{x} u_{x x}\right)+3 \mu \alpha^{2} u^{2} u_{x}=0
\end{aligned}
$$

is also an important physical model. The above two equations were studied by many authors. Equation (2) was introduced by Fuchssteiner and Fokas in their previous works [41, 42] in 1981. The Lax pairs of (2) were given by Fokas in [40]. New Lax pairs and Darboux transformation of (2) were introduced by Yang and Rui in [43] recently. In [44], by using the bifurcation theory of dynamical system, the existence conditions of different kinds of traveling wave solutions of (2) were presented by Bi. In [45], by using the same method, Li and Zhang studied (1), the existence of solitary wave, kink and antikink wave solutions, uncountably infinite many smooth, and nonsmooth periodic wave solutions were discussed. However, exact travelling wave solutions of (1) were not obtained in [45] though the authors obtained some results of numerical simulation for smooth and nonsmooth periodic wave solutions in this work. Moreover, the investigations on exact solutions of (1) are few in the existing literatures because (1) is more complex than (2). Therefore, in this paper, employing Frobenius' idea together with integral bifurcation method, we will investigate different kinds of exact traveling wave solutions of (1).

The rest of this paper is organized as follows. In Section 2, by using Frobenius' idea, we will derive ordinary differential equation (ODE) which is equivalent to (1). In Section 3, by using the integral bifurcation method combined with factoring technique, we will investigate different kinds of exact traveling wave solutions of (1) and discuss their dynamic properties when the integral constants satisfy different conditions. In Section 4, we will discuss different kinds of exact traveling wave solutions of (1) under the special case of the parameter $v=0$.

\section{Direct Application of Frobenius' Idea on Reducing the PDE (1) to an Integrable ODE}

Frobenius' idea is about changing a partial differential equation (PDE) into an ordinary differential equation (ODE) and then using integrable decomposition method to investigate its exact solutions. Thus, in this section, we first employ the direct Frobenius' idea to change (1) into an integrable ordinary differential equation; see the following discussions.

Making a traveling wave transformation $u(x, t)=u(\xi)$ with $\xi=x-c t$, (1) can be reduced to the following ordinary differential equation (ODE):

$$
\begin{aligned}
&(1-c) \frac{d u}{d \xi}+(\beta-c \nu) \frac{d^{3} u}{d \xi^{3}}+\alpha u \frac{d u}{d \xi} \\
&+ \frac{1}{3} \nu \alpha\left(u \frac{d^{3} u}{d \xi^{3}}+2 \frac{d u}{d \xi} \frac{d^{2} u}{d \xi^{2}}\right)+3 \mu \alpha^{2} u^{2} \frac{d u}{d \xi} \\
&+\nu \mu \alpha^{2}\left[u^{2} \frac{d^{3} u}{d \xi^{3}}+\left(\frac{d u}{d \xi}\right)^{3}+4 u \frac{d u}{d \xi} \frac{d^{2} u}{d \xi^{2}}\right] \\
&+\mu \nu^{2} \alpha^{2}\left[\left(\frac{d u}{d \xi}\right)^{2} \frac{d^{3} u}{d \xi^{3}}+2 \frac{d u}{d \xi}\left(\frac{d^{2} u}{d \xi^{2}}\right)^{2}\right]=0,
\end{aligned}
$$

where $c$ is wave velocity which moves along the direction of $x$-axis and $c \neq 0$. Equation (3) can be rewritten as

$$
\begin{aligned}
& (1-c) \frac{d u}{d \xi}+(\beta-c \nu) \frac{d^{3} u}{d \xi^{3}}+\frac{1}{2} \alpha \frac{d\left(u^{2}\right)}{d \xi} \\
& +\frac{1}{3} \nu \alpha \frac{d}{d \xi}\left[u \frac{d^{2} u}{d \xi^{2}}+\frac{1}{2}\left(\frac{d u}{d \xi}\right)^{2}\right] \\
& +\mu \alpha^{2} \frac{d\left(u^{3}\right)}{d \xi}+\nu \mu \alpha^{2} \frac{d}{d \xi}\left[u^{2} \frac{d^{2} u}{d \xi^{2}}+u\left(\frac{d u}{d \xi}\right)^{2}\right] \\
& +\mu \nu^{2} \alpha^{2} \frac{d}{d \xi}\left[\left(\frac{d u}{d \xi}\right)^{2} \frac{d^{2} u}{d \xi^{2}}\right]=0 .
\end{aligned}
$$

Integrating (4) once, we obtain

$$
\begin{aligned}
& (1-c) u+(\beta-c \nu) \frac{d^{2} u}{d \xi^{2}}+\frac{1}{2} \alpha u^{2} \\
& +\frac{1}{3} \nu \alpha\left[u \frac{d^{2} u}{d \xi^{2}}+\frac{1}{2}\left(\frac{d u}{d \xi}\right)^{2}\right] \\
& +\mu \alpha^{2} u^{3}+\nu \mu \alpha^{2}\left[u^{2} \frac{d^{2} u}{d \xi^{2}}+u\left(\frac{d u}{d \xi}\right)^{2}\right] \\
& +\mu \nu^{2} \alpha^{2}\left[\left(\frac{d u}{d \xi}\right)^{2} \frac{d^{2} u}{d \xi^{2}}\right]=g,
\end{aligned}
$$

where $g$ is an integral constant. Employing direct Frobenius' idea, we need not change (5) into a 2-dimensional planar system as the method in [33-36]. we can directly integrate (5) again; see the following calculus. 
Multiplying $d u / d \xi$ to the both sides of (5) yields

$$
\begin{aligned}
(1-c) & u \frac{d u}{d \xi}+(\beta-c \nu) \frac{d u}{d \xi} \frac{d^{2} u}{d \xi^{2}}+\frac{1}{2} \alpha u^{2} \frac{d u}{d \xi} \\
+ & \frac{1}{3} \nu \alpha\left[u \frac{d u}{d \xi} \frac{d^{2} u}{d \xi^{2}}+\frac{1}{2}\left(\frac{d u}{d \xi}\right)^{3}\right] \\
+ & \mu \alpha^{2} u^{3} \frac{d u}{d \xi}+\nu \mu \alpha^{2}\left[u^{2} \frac{d u}{d \xi} \frac{d^{2} u}{d \xi^{2}}+u\left(\frac{d u}{d \xi}\right)^{3}\right] \\
+ & \mu \nu^{2} \alpha^{2}\left[\left(\frac{d u}{d \xi}\right)^{3} \frac{d^{2} u}{d \xi^{2}}\right]=g \frac{d u}{d \xi}
\end{aligned}
$$

Integrating (6) once, we obtain

$$
\begin{aligned}
-g u & +\frac{1}{2}(1-c) u^{2}+\frac{1}{2}(\beta-c \nu)\left(\frac{d u}{d \xi}\right)^{2}+\frac{1}{6} \alpha u^{3} \\
& +\frac{1}{6} \nu \alpha u\left(\frac{d u}{d \xi}\right)^{2}+\frac{1}{4} \mu \alpha^{2} u^{4}+\frac{1}{4} \nu \mu^{2} \alpha^{2}\left(\frac{d u}{d \xi}\right)^{4} \\
& +\frac{1}{2} \nu \mu \alpha^{2} u^{2}\left(\frac{d u}{d \xi}\right)^{2}=h,
\end{aligned}
$$

where $h$ is another arbitrary integral constant. When $v \neq 0$, (7) can be rewritten as

$$
\begin{gathered}
-\frac{4 h}{v}-\frac{4 g}{v} u+\frac{2(1-c)}{v} u^{2}+\frac{2 \alpha}{3 v} u^{3}+\frac{\mu \alpha^{2}}{v} u^{4} \\
+\frac{2(\beta-c \nu)}{v}\left(\frac{d u}{d \xi}\right)^{2}+\frac{2 \alpha}{3} u\left(\frac{d u}{d \xi}\right)^{2} \\
+2 \mu \alpha^{2} u^{2}\left(\frac{d u}{d \xi}\right)^{2}+\mu^{2} \alpha^{2}\left(\frac{d u}{d \xi}\right)^{4}=0 .
\end{gathered}
$$

\section{Different Kinds of Exact Traveling Wave Solutions of (1)}

In this section, by using the integral bifurcation method combined with factoring technique as in [36], we will investigate different kinds of exact traveling wave solutions of (1) and discuss their dynamic properties via (7) and (8).

3.1. Hyperbolic Function Solutions and Periodic Wave Solutions of (1) as the Two Integral Constants $g \neq 0$ and $h \neq 0$. When $\nu(\nu-\mu)>0$ and $h=\left(324 \nu \mu^{2}(c-1)^{2}+\mu\left(648 c \nu^{2}-648 \nu \beta+\right.\right.$ $\left.\left.324 \beta^{2}+36 c \nu-36 \nu-324 c^{2} \nu^{2}\right)+\nu+36 \nu(\beta-c \nu)\right) / 1296 v(\mu-$ v) $\alpha^{2} \mu^{2} \neq 0, g=(18 \mu(c-1)+1) / 108 \alpha \mu^{2} \neq 0$, (8) can be decomposed in the following form:

$$
\begin{aligned}
{\left[r_{0}+\right.} & \left.r_{1} u+r_{2} u^{2}+\mu^{2}\left(\frac{d u}{d \xi}\right)^{2}\right] \\
& \times\left[s_{0}+s_{1} u+s_{2} u^{2}+\alpha^{2}\left(\frac{d u}{d \xi}\right)^{2}\right]=0,
\end{aligned}
$$

where the coefficients $r_{0}, r_{1}, r_{2}, s_{0}, s_{1}$, and $s_{2}$ are defined by the following expressions:

$$
\begin{gathered}
r_{0}=\frac{\nu[c \nu-\beta+\mu(1-c)-1 / 18]-(c \nu-\beta) \sqrt{\nu(\nu-\mu)}}{\nu \alpha^{2} \sqrt{\nu(\nu-\mu)}}, \\
r_{1}=\frac{\mu-\nu+\sqrt{\nu(\nu-\mu)}}{3 \alpha \sqrt{\nu(\nu-\mu)}}, \quad r_{2}=1-\frac{\mu}{\nu} \sqrt{\nu(\nu-\mu)}, \\
s_{0}=-\frac{\nu[c \nu-\beta+\mu(1-c)-1 / 18]+(c \nu-\beta) \sqrt{\nu(\nu-\mu)}}{\nu \mu^{2} \sqrt{\nu(\nu-\mu)}}, \\
s_{1}=\frac{\alpha[\nu-\mu+\sqrt{\nu(\nu-\mu)}]}{3 \mu^{2} \sqrt{\nu(\nu-\mu)}}, \quad s_{2}=\frac{\alpha^{2}}{\mu}-\frac{\alpha^{2}}{\mu \nu} \sqrt{\nu(\nu-\mu)} .
\end{gathered}
$$

Thus (9) can be reduced to the following two ordinary differential equations:

$$
r_{0}+r_{1} u+r_{2} u^{2}+\mu^{2}\left(\frac{d u}{d \xi}\right)^{2}=0
$$

or

$$
s_{0}+s_{1} u+s_{2} u^{2}+\alpha^{2}\left(\frac{d u}{d \xi}\right)^{2}=0
$$

where the coefficients $r_{0}, r_{1}, r_{2}, s_{0}, s_{1}$, and $s_{2}$ are given by (10).

Solving (11) under the conditions $r_{2}<0$ and $q=4 r_{0} r_{2}-$ $r_{1}^{2}>0$, we obtain two hyperbolic function solutions of (1) as follows:

$$
u= \pm \frac{1}{2 r_{2}}\left[\sqrt{q} \sinh \left(\frac{\sqrt{-r_{2}}}{\mu}(x-c t)\right) \mp r_{1}\right] .
$$

Solving (11) under the conditions $r_{2}>0$ and $q=4 r_{0} r_{2}-$ $r_{1}^{2}<0$, we obtain two periodic wave solutions of (1) as follows:

$$
u=\mp \frac{1}{2 r_{2}}\left[\sqrt{-q} \sin \left(\frac{\sqrt{r_{2}}}{\mu}(x-c t)\right) \pm r_{1}\right] \text {. }
$$

Similarly, solving (12) under the conditions $s_{2}<0$ and $\widetilde{q}=$ $4 s_{0} s_{2}-s_{1}^{2}>0$, we obtain two hyperbolic function solutions of (1) as follows:

$$
u= \pm \frac{1}{2 s_{2}}\left[\sqrt{\widetilde{q}} \sinh \left(\frac{\sqrt{-s_{2}}}{\alpha}(x-c t)\right) \mp s_{1}\right] .
$$

Solving (12) under the conditions $s_{2}>0$ and $\tilde{q}=4 s_{0} s_{2}-$ $s_{1}^{2}<0$, we obtain two periodic wave solutions of (1) as follows:

$$
u=\mp \frac{1}{2 s_{2}}\left[\sqrt{-\tilde{q}} \sin \left(\frac{\sqrt{s_{2}}}{\alpha}(x-c t)\right) \pm s_{1}\right] .
$$


3.2. Hyperbolic Function Solutions and Periodic Wave Solutions of (1) as the Two Integral Constants $g=0$ and $h \neq 0$. When $\nu^{2}-\mu \nu>0$ and $g=0, h=(18 \mu \nu-18 \mu \beta-\nu)^{2} /$ $1296 \nu \alpha^{2} \mu^{3}(\nu-\mu) \neq 0, c=1-(1 / 18 \mu)$, (8) can be decomposed in the following form:

$$
\begin{gathered}
{\left[\frac{\mu \nu-\mu \beta-v / 18}{\nu \mu^{2}\left(\nu-\mu+\sqrt{\nu^{2}-\mu \nu}\right)}+\frac{\alpha\left(\mu-\nu+\sqrt{\nu^{2}-\mu \nu}\right)}{3 \mu^{2} \sqrt{\nu^{2}-\mu \nu}} u\right.} \\
\left.+\frac{\alpha^{2}}{\mu}\left(1-\frac{\sqrt{\nu^{2}-\mu \nu}}{\nu}\right) u^{2}+\alpha^{2}\left(\frac{d u}{d \xi}\right)^{2}\right] \\
\times\left[\frac{\mu \nu-\mu \beta-v / 18}{\nu \alpha^{2}\left(\nu-\mu-\sqrt{\nu^{2}-\mu \nu}\right)}+\frac{2-\mu+\sqrt{\nu^{2}-\mu \nu}}{3 \alpha \sqrt{\nu^{2}-\mu \nu}} u\right. \\
\left.+\left(\mu+\frac{\mu}{\nu} \sqrt{\nu^{2}-\mu \nu}\right) u^{2}+\mu^{2}\left(\frac{d u}{d \xi}\right)^{2}\right]=0 .
\end{gathered}
$$

Equation (17) can be reduced to the following two ordinary differential equations:

$$
\begin{gathered}
{\left[\frac{\mu \nu-\mu \beta-\nu / 18}{\nu \mu^{2}\left(\nu-\mu+\sqrt{\nu^{2}-\mu \nu}\right)}+\frac{\alpha\left(\mu-\nu+\sqrt{\nu^{2}-\mu \nu}\right)}{3 \mu^{2} \sqrt{\nu^{2}-\mu \nu}} u\right.} \\
\left.\quad+\frac{\alpha^{2}}{\mu}\left(1-\frac{\sqrt{\nu^{2}-\mu \nu}}{\nu}\right) u^{2}+\alpha^{2}\left(\frac{d u}{d \xi}\right)^{2}\right]=0
\end{gathered}
$$

or

$$
\begin{gathered}
{\left[\frac{\mu \nu-\mu \beta-\nu / 18}{\nu \alpha^{2}\left(\nu-\mu-\sqrt{\nu^{2}-\mu \nu}\right)}+\frac{\nu-\mu+\sqrt{\nu^{2}-\mu \nu}}{3 \alpha \sqrt{\nu^{2}-\mu \nu}} u\right.} \\
\left.+\left(\mu+\frac{\mu}{v} \sqrt{\nu^{2}-\mu \nu}\right) u^{2}+\mu^{2}\left(\frac{d u}{d \xi}\right)^{2}\right]=0 .
\end{gathered}
$$

Solving (18), we obtain two hyperbolic function solutions and two periodic wave solutions of (1) as follows:

$$
\begin{aligned}
u= & \pm \frac{\mu \nu}{2 \alpha^{2}\left(\nu-\sqrt{\nu^{2}-\mu \nu}\right)} \\
& \times\left[\frac{\alpha \Omega_{1}}{3 \mu \nu} \sinh \left(\omega_{1} \xi\right) \mp \frac{\alpha\left(\mu-\nu+\sqrt{\nu^{2}-\mu \nu}\right)}{3 \mu^{2} \sqrt{\nu^{2}-\mu \nu}}\right],
\end{aligned}
$$

$$
\begin{aligned}
u= & \mp \frac{\mu \nu}{2 \alpha^{2}\left(\nu-\sqrt{\nu^{2}-\mu \nu}\right)} \\
& \times\left[\frac{\alpha \Omega_{2}}{3 \mu \nu} \sin \left(\omega_{2} \xi\right) \pm \frac{\alpha\left(\mu-\nu+\sqrt{\nu^{2}-\mu \nu}\right)}{3 \mu^{2} \sqrt{\nu^{2}-\mu \nu}}\right],
\end{aligned}
$$

where the $\Omega_{1}, \Omega_{2}, \omega_{1}, \omega_{2}$, and $\xi$ are defined by

$$
\begin{gathered}
\Omega_{1}=\sqrt{\frac{[36 \mu(\beta-\nu)+\nu] \sqrt{\nu^{2}-\mu \nu}+\left[36 \mu \nu(\nu-\beta)+\nu^{2}-\mu \nu\right]}{\mu\left(\nu-\mu+\sqrt{\nu^{2}-\mu \nu}\right)}}, \\
\omega_{1}=\sqrt{\frac{\sqrt{\nu^{2}-\mu \nu}-\nu}{\mu \nu}},
\end{gathered}
$$

$\Omega_{2}$

$$
\begin{gathered}
=\sqrt{-\frac{[36 \mu(\beta-\nu)+\nu] \sqrt{\nu^{2}-\mu \nu}+\left[36 \mu \nu(\nu-\beta)+\nu^{2}-\mu \nu\right]}{\mu\left(\nu-\mu+\sqrt{\nu^{2}-\mu \nu}\right)}}, \\
\omega_{2}=\sqrt{\frac{\nu-\sqrt{\nu^{2}-\mu \nu}}{\mu \nu}}, \quad \xi=x-\left(1-\frac{1}{18 \mu}\right) t .
\end{gathered}
$$

Similarly solving (19), we also obtain two hyperbolic function solutions and two periodic wave solutions of (1) as follows:

$$
\begin{aligned}
u= & \pm \frac{v}{2 \mu\left(\nu+\sqrt{\nu^{2}-\mu \nu}\right)} \\
& \times\left[\frac{\mu \Omega_{3}}{3 \alpha \nu} \sinh \left(\omega_{3} \xi\right) \mp \frac{\nu-\mu+\sqrt{\nu^{2}-\mu \nu}}{3 \alpha \sqrt{\nu^{2}-\mu \nu}}\right], \\
u=\mp \frac{\nu}{2 \mu\left(\nu+\sqrt{\nu^{2}-\mu \nu}\right)} & \\
& \times\left[\frac{\mu \Omega_{4}}{3 \alpha \nu} \sin \left(\omega_{4} \xi\right) \pm \frac{\nu-\mu+\sqrt{\nu^{2}-\mu \nu}}{3 \alpha \sqrt{\nu^{2}-\mu \nu}}\right],
\end{aligned}
$$

where the $\Omega_{3}, \Omega_{4}, \omega_{3}, \omega_{4}$, and $\xi$ are defined by

$\Omega_{3}$

$$
\begin{aligned}
& =\sqrt{\frac{[36(\nu-\beta)-\nu] \sqrt{\nu^{2}-\mu \nu}+\left[36 \nu(\nu-\beta)-\nu^{2} / \mu-\nu\right]}{\nu-\mu-\sqrt{\nu^{2}-\mu \nu}}}, \\
& \omega_{3}=\frac{1}{\mu} \sqrt{-\left(\mu+\frac{\mu}{\nu} \sqrt{\nu^{2}-\mu \nu}\right)},
\end{aligned}
$$


$\Omega_{4}$

$$
\begin{gathered}
=\sqrt{-\frac{[36(\nu-\beta)-\nu] \sqrt{\nu^{2}-\mu \nu}+\left[36 \nu(\nu-\beta)-v^{2} / \mu-\nu\right]}{\nu-\mu-\sqrt{\nu^{2}-\mu \nu}}}, \\
\omega_{4}=\frac{1}{\mu} \sqrt{\mu+\frac{\mu}{\nu} \sqrt{\nu^{2}-\mu \nu}}, \quad \xi=x-\left(1-\frac{1}{18 \mu}\right) t .
\end{gathered}
$$

3.3. Hyperbolic Function Solutions and Periodic Wave Solutions of (1) as the Two Integral Constants $g \neq 0$ and $h=0$. When $\nu^{2}-\mu \nu>0$ and $g=(\nu+18 \mu(\beta-\nu)) / 108 \alpha \mu^{2} \sqrt{\nu^{2}-\mu \nu} \neq$ $0, h=0, c=\left(\nu+18 \nu(\beta-\mu)+18 \sqrt{\nu^{2}-\mu \nu}\right) / 18 \nu(\nu-\mu+$ $\left.\sqrt{\nu^{2}-\mu \nu}\right),(8)$ can be decomposed in the following form:

$$
\begin{aligned}
& {\left[\frac{18 \mu(\nu-\beta)-v}{9 v \mu^{2}\left(\nu-\mu+\sqrt{\nu^{2}-\mu \nu}\right)}+\frac{\alpha\left(\mu-\nu+\sqrt{\nu^{2}-\mu \nu}\right)}{3 \mu^{2} \sqrt{\nu^{2}-\mu \nu}} u\right.} \\
& \left.+\frac{\alpha^{2}\left(\nu-\sqrt{\nu^{2}-\mu \nu}\right)}{\nu \mu} u^{2}+\alpha^{2}\left(\frac{d u}{d \xi}\right)^{2}\right] \\
& \times\left[\frac{v-\mu+\sqrt{\nu^{2}-\mu \nu}}{3 \alpha \sqrt{\nu^{2}-\mu \nu}} u+\left(1+\frac{\mu}{v} \sqrt{v^{2}-\mu \nu}\right) u^{2}\right. \\
& \left.+\mu^{2}\left(\frac{d u}{d \xi}\right)^{2}\right]=0 .
\end{aligned}
$$

Similarly, solving (26) we obtain four hyperbolic function solutions and four periodic wave solutions of (1) as follows:

$$
\begin{aligned}
u= & \pm \frac{\mu \nu}{2 \alpha^{2}\left(\nu-\sqrt{\nu^{2}-\mu \nu}\right)} \\
& \times\left[\frac{\alpha \Omega_{5}}{3 \mu \nu} \sinh \left(\omega_{5} \xi\right) \pm \frac{\alpha\left(\nu-\mu-\sqrt{\nu^{2}-\mu \nu}\right)}{3 \mu^{2} \sqrt{\nu^{2}-\mu \nu}}\right], \\
u= & \left.\frac{\mu \nu}{2 \alpha^{2}\left(\nu-\sqrt{\nu^{2}-\mu \nu}\right)}\right] \\
& \times\left[\frac{\alpha \Omega_{6}}{3 \mu \nu} \sin \left(\omega_{6} \xi\right) \mp \frac{\alpha\left(\nu-\mu-\sqrt{\nu^{2}-\mu \nu}\right)}{3 \mu^{2} \sqrt{\nu^{2}-\mu \nu}}\right],
\end{aligned}
$$

where the $\Omega_{5}, \Omega_{6}, \omega_{5}, \omega_{6}$, and $\xi$ are defined by

$$
\begin{gathered}
\Omega_{5} \sqrt{\frac{72 \mu \nu(\nu-\beta)-\left(3 \nu^{2}-\mu \nu\right)+[72 \mu(\beta-\nu)+3 \nu] \sqrt{\nu^{2}-\mu \nu}}{\mu\left(\nu-\mu+\sqrt{\nu^{2}-\mu \nu}\right)}}, \\
\omega_{5}=\sqrt{\frac{\nu-\sqrt{\nu^{2}-\mu \nu}}{\mu \nu}},
\end{gathered}
$$

$\Omega_{6}$

$$
\begin{aligned}
& =\sqrt{\frac{72 \mu \nu(\nu-\beta)-\left(3 \nu^{2}-\mu \nu\right)+[72 \mu(\beta-\nu)+3 \nu] \sqrt{\nu^{2}-\mu \nu}}{\mu\left(\nu-\mu+\sqrt{\nu^{2}-\mu \nu}\right)}}, \\
& \omega_{6}=\sqrt{\frac{\sqrt{\nu^{2}-\mu \nu}-v}{\mu \nu}}, \\
& \xi=x-\frac{\nu+18 \nu(\beta-\mu)+18 \sqrt{\nu^{2}-\mu \nu}}{18 \nu\left(\nu-\mu+\sqrt{\nu^{2}-\mu \nu}\right)} t, \\
& u= \pm \frac{\nu^{2}-\mu \nu+\nu \sqrt{\nu^{2}-\mu \nu}}{6 \alpha \mu\left(\nu+\sqrt{\nu^{2}-\mu \nu}\right) \sqrt{\nu^{2}-\mu \nu}} \\
& \times\left[\sinh \left(\sqrt{-\frac{\nu+\sqrt{\nu^{2}-\mu \nu}}{\mu \nu}} \xi\right) \mp 1\right] \text {, } \\
& u=\mp \frac{v^{2}-\mu \nu+\nu \sqrt{\nu^{2}-\mu \nu}}{6 \alpha \mu\left(\nu+\sqrt{\nu^{2}-\mu \nu}\right) \sqrt{\nu^{2}-\mu \nu}} \\
& \times\left[\sin \left(\sqrt{\frac{\nu+\sqrt{\nu^{2}-\mu \nu}}{\mu \nu}} \xi\right) \pm 1\right],
\end{aligned}
$$

where $\xi=x-\left(\left(\nu+18 \nu(\beta-\mu)+18 \sqrt{\nu^{2}-\mu \nu}\right) / 18 \nu(\nu-\mu+\right.$ $\left.\sqrt{\nu^{2}-\mu \nu}\right) t$.

3.4. Hyperbolic Function Solutions, Periodic Wave Solutions, and Rational Function Solution of (1) as the Two Integral Constants $g=h=0$. (i) When $\mu=\nu, \beta>\nu$ and $g=h=0$, $c=\beta / \nu,(8)$ can be decomposed in the following form:

$$
\begin{aligned}
& {\left[\frac{\alpha}{3 v^{2}}(1+\sqrt{1+18(\beta-v)}) u+\frac{\alpha^{2}}{\nu} u^{2}+\alpha^{2}\left(\frac{d u}{d \xi}\right)^{2}\right]} \\
& \quad \times\left[\frac{1-\sqrt{1+18(\beta-v)}}{3 \alpha} u+v u^{2}+\mu^{2}\left(\frac{d u}{d \xi}\right)^{2}\right]=0 .
\end{aligned}
$$


Solving (32) we obtain four hyperbolic function solutions and four periodic wave solutions of (1) as follows:

$$
\begin{aligned}
& u= \pm \frac{1+\sqrt{1+18(\beta-\nu)}}{6 \alpha \nu}\left[\sinh \left(\frac{\xi}{\sqrt{-\nu}}\right) \mp 1\right] \\
& (\nu<0<\beta), \\
& u=\mp \frac{1+\sqrt{1+18(\beta-\nu)}}{6 \alpha \nu}\left[\sin \left(\frac{\xi}{\sqrt{\nu}}\right) \pm 1\right], \quad(\beta>v>0), \\
& u=\mp \frac{1+\sqrt{1+18(\beta-\nu)}}{6 \alpha \nu}\left[\sinh \left(\frac{\sqrt{-\nu} \xi}{\mu}\right) \pm 1\right] \\
& (\nu<0<\beta), \\
& u= \pm \frac{1+\sqrt{1+18(\beta-v)}}{6 \alpha \nu}\left[\sin \left(\frac{\sqrt{\nu} \xi}{\mu}\right) \mp 1\right] \\
& (\beta>v>0) \text {, }
\end{aligned}
$$

where $\xi=x-(\beta / \nu) t$.

(ii) When $\mu=-v / 18(\beta-v), \beta>\nu$ and $g=h=0, c=\beta / \nu$, (8) can be decomposed in the following form:

$$
\begin{aligned}
{[A u} & \left.-\frac{\alpha \nu A}{6(\beta-v)} u^{2}+\alpha^{2}\left(\frac{d u}{d \xi}\right)^{2}\right] \\
& \times\left[B u-\frac{v B}{6(\beta-v)} u^{2}+\mu^{2}\left(\frac{d u}{d \xi}\right)^{2}\right]=0,
\end{aligned}
$$

where $A=6 \alpha(\beta-\nu)\left[18(\beta-\nu)-3 \sqrt{36(\beta-\nu)^{2}+2(\beta-\nu)}\right] / \nu^{2}$, $B=\left(18(\beta-\nu)+3 \sqrt{36(\beta-\nu)^{2}+2(\beta-\nu)}\right) / 54 \alpha(\beta-\nu)$.

Solving (37) we obtain two hyperbolic function solutions and two periodic wave solutions of (1) as follows:

$$
\begin{array}{r}
u=\frac{6(\beta-v)}{\alpha \nu} \cos ^{2}\left(\frac{1}{2} \sqrt{\frac{\nu A}{6 \alpha(\nu-\beta)}} \xi\right), \\
(0<\beta<\nu, A>0), \\
u=\frac{6(\beta-v)}{\alpha \nu} \cosh ^{2}\left(\frac{1}{2} \sqrt{\frac{v A}{6 \alpha(\beta-\nu)}} \xi\right), \\
(v<0<\beta, A<0),
\end{array}
$$

$$
\begin{array}{r}
u=\frac{6(\beta-v)}{v} \cos ^{2}\left(\frac{1}{2 \mu} \sqrt{\frac{\nu B}{6(\nu-\beta)}} \xi\right), \\
(0<\beta<\nu, B>0), \\
u=\frac{6(\beta-v)}{v} \cosh ^{2}\left(\frac{1}{2 \mu} \sqrt{\frac{v B}{6(\beta-\nu)}} \xi\right), \\
(\beta>v>0, B>0),
\end{array}
$$

where $\xi=x-(\beta / v) t$ and $A, B$ have been given above.

(iii)When $\mu=0$ and $g=h=0, c=\beta / \nu$, (8) can be decomposed in the following form:

$$
\begin{gathered}
{\left[\frac{3 \alpha(v-\beta)}{v^{2}} u+\frac{\alpha^{2}}{v} u^{2}+\alpha^{2}\left(\frac{d u}{d \xi}\right)^{2}\right]} \\
\times\left[\frac{2}{3 \alpha} u+\mu^{2}\left(\frac{d u}{d \xi}\right)^{2}\right]=0 .
\end{gathered}
$$

Solving (42), we obtain a hyperbolic function solution, a periodic wave solution, and a rational function solution as follows:

$$
\begin{gathered}
u=\frac{3(\beta-\nu)}{\alpha \nu} \cos ^{2}\left(\frac{1}{2 \sqrt{v}} \xi\right), \quad(\nu>0), \\
u=\frac{3(\beta-v)}{\alpha \nu} \cosh ^{2}\left(\frac{1}{2 \sqrt{-v}} \xi\right), \quad(\nu<0), \\
u=-\frac{1}{6 \alpha \mu^{2}} \xi^{2},
\end{gathered}
$$

where $\xi=x-(\beta / \nu) t$ and $A, B$ have been given above.

All the above exact solutions which were obtained by us are smooth travelling wave solutions including smooth periodic wave solutions and smooth hyperbolic function solutions. In order to show the dynamical profiles of periodic wave solutions, as examples, we plot the graphs of solutions (14) and (38) for $\alpha=0.5, \beta=0.8, \mu=1.2, t=0.1$, which are shown in Figures 1(a) and 1(b).

3.5. Peakon Solutions under Some Special Parametric Condition. The expression in the right side of (8) cannot be reduced to a form of $\left[a u^{2}-b(d u / d \xi)^{2}\right]^{2}=0$ by using the factoring technique because this equation contains the terms $-(4 h / v)-$ $(4 g / v) u+(2 \alpha / 3 v) u^{3}+(2 \alpha / 3) u(d u / d \xi)^{2}$. Thus, the peakon solutions of (1) such as Cammasa-Holm's form $r e^{-\delta|\xi|}$ cannot be obtained by direct integral method together with factoring technique as in [36]. However, the research works given by $\mathrm{Li}$ et al. in [45] show that the peakon solutions of (1) exist though they did not obtain exact peakon solutions of this equation. Indeed, the existence of peakon solution of (1) is proved by $\mathrm{Li}$ via analysis of phase portraits in this paper. We notice that the terms $u^{3}$ and $u(d u / d \xi)^{2}$ are kindred terms when $u=r e^{-\delta|\xi|}$. Thus we assume that (1) has peakon solutions of the form $u=r e^{-\delta|\xi|}$ or $u=s+r e^{-\delta|\xi|}$. 


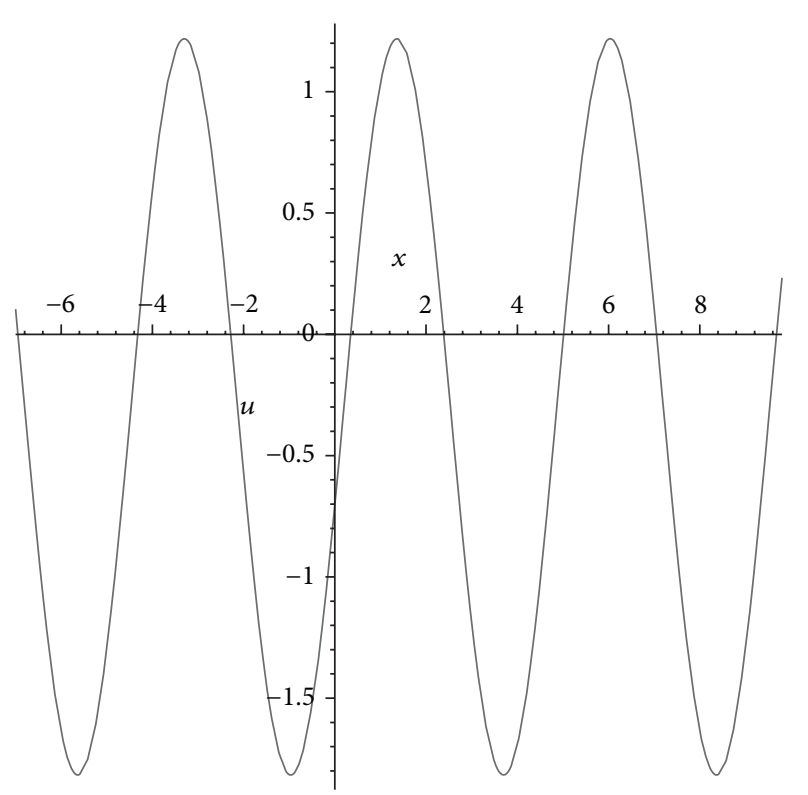

(a) Periodic wave defined by (14)

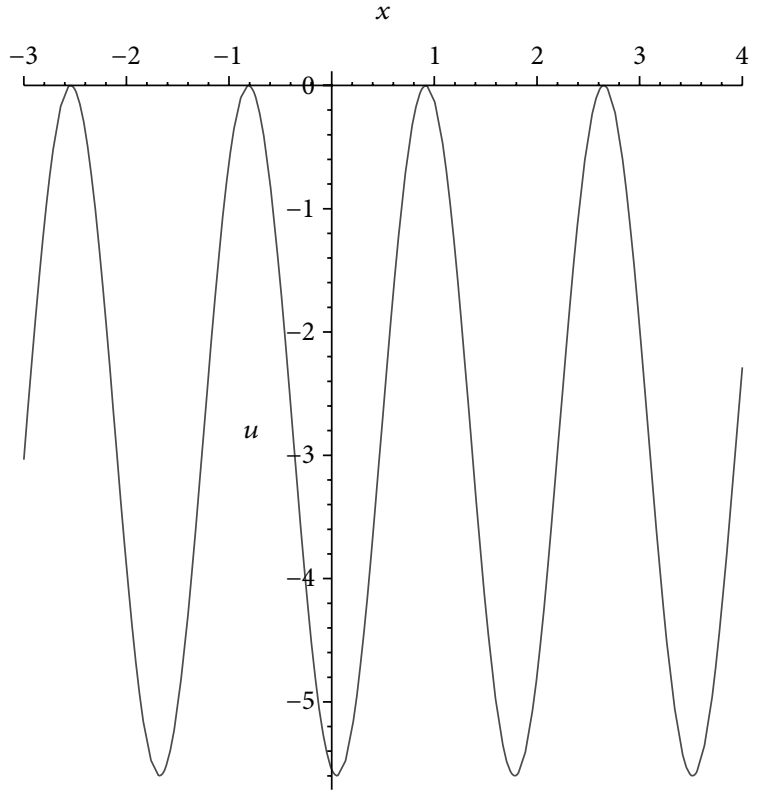

(b) Periodic wave defined by (38)

Figure 1: The graphs of profiles for the smooth periodic wave solutions defined by (14).

(a) When $g \neq 0$ and $h \neq 0$, we suppose that (8) has a peakon solution as the following form:

$$
u=s+r e^{-\delta|\xi|},
$$

where the parameters $s, r$, and $\delta$ can be determined further in the below discussions. We define $s+r e^{-\delta|\xi|}=s+r \exp \left(-\delta \sqrt{\xi^{2}}\right)$ when $\xi \neq 0$; particularly $u=s+r=$ (constant) when $\xi=0$, and obviously $u=s+r$ is a constant solution which satisfies (1).

When $\xi \neq 0$, substituting (46) (i.e. $u=s+r \exp \left(-\delta \sqrt{\xi^{2}}\right)$ ) into (8) we obtain

$$
\begin{aligned}
C_{0}+ & C_{1} \exp \left(-\delta \sqrt{\xi^{2}}\right)+C_{2} \exp \left(-2 \delta \sqrt{\xi^{2}}\right) \\
& +C_{3} \exp \left(-3 \delta \sqrt{\xi^{2}}\right)+C_{4} \exp \left(-4 \delta \sqrt{\xi^{2}}\right)=0,
\end{aligned}
$$

where coefficients $C_{0}, C_{1}, C_{2}, C_{3}$, and $C_{4}$ satisfy

$$
\begin{aligned}
C_{0}= & -\frac{4 h}{v}-\frac{4 g s}{v}+\frac{2 \alpha s^{3}}{3 v}+\frac{2(1-c) s^{2}}{v}+\frac{\mu \alpha^{2} s^{4}}{v}, \\
C_{1}= & \frac{4(1-c) r s}{v}+\frac{2 \alpha r s}{v}-\frac{4 g r}{v}+\frac{4 \mu \alpha^{2} r s^{3}}{v}, \\
C_{2}= & \frac{2(1-c) r^{2}}{v}+\frac{2 \alpha r^{2} s}{v}+\frac{6 \mu \alpha^{2} r^{2} s^{2}}{v} \\
& +2 \mu \alpha^{2} r^{2} s^{2} \delta^{2}+\frac{2(\beta-c \nu) r^{2} \delta^{2}}{v}+\frac{2 \alpha r^{2} s \delta^{2}}{3},
\end{aligned}
$$

$$
\begin{aligned}
& C_{3}=\frac{\mu \alpha^{2} r^{4}}{\nu}+2 \mu \alpha^{2} r^{4} \delta^{2}+\mu^{2} \alpha^{2} r^{4} \delta^{4}, \\
& C_{4}=\frac{\mu \alpha^{2} r^{4}+2 \mu \alpha^{2} r^{4} \delta^{2}+\mu^{2} \alpha^{2} r^{4} \delta^{4}}{\nu} .
\end{aligned}
$$

Let the coefficients of every terms of exp-function (including the term of constant) in (47) as zero; it follows

$$
C_{0}=0, \quad C_{1}=0, \quad C_{2}=0, \quad C_{3}=0, \quad C_{4}=0 .
$$

Solving the above group of equations yields

$$
c=\frac{\left(\nu^{2}-3 \mu \nu+36 \mu^{2} \nu-36 \beta \mu \nu\right)+(\nu-36 \beta \mu) \sqrt{\nu^{2}-\mu \nu}}{36 \mu \nu\left(\mu-\nu-\sqrt{\nu^{2}-\mu \nu}\right)},
$$

$$
=-\frac{\left(\nu^{2}+\mu \nu-36 \mu \nu^{2}+36 \beta \mu \nu\right)+(\nu+36 \beta \mu-36 \mu \nu) \sqrt{\nu^{2}-\mu \nu}}{216 \mu^{2} \nu\left(\mu-\nu-\sqrt{\nu^{2}-\mu \nu}\right)},
$$

$h$

$$
\begin{gathered}
=-\frac{\left(3 \nu^{2}+\mu \nu-72 \mu \nu^{2}+72 \beta \mu \nu\right)+(3 \nu+72 \beta \mu-72 \mu \nu) \sqrt{\nu^{2}-\mu \nu}}{5184 \mu^{3} \nu \alpha^{2}\left(\mu-\nu-\sqrt{\nu^{2}-\mu \nu}\right)}, \\
r=r(\text { free parameter }), \quad s=-\frac{1}{6 \alpha \mu}, \\
\delta=\sqrt{\frac{\sqrt{\nu^{2}-\mu \nu}-v}{\mu \nu}},
\end{gathered}
$$




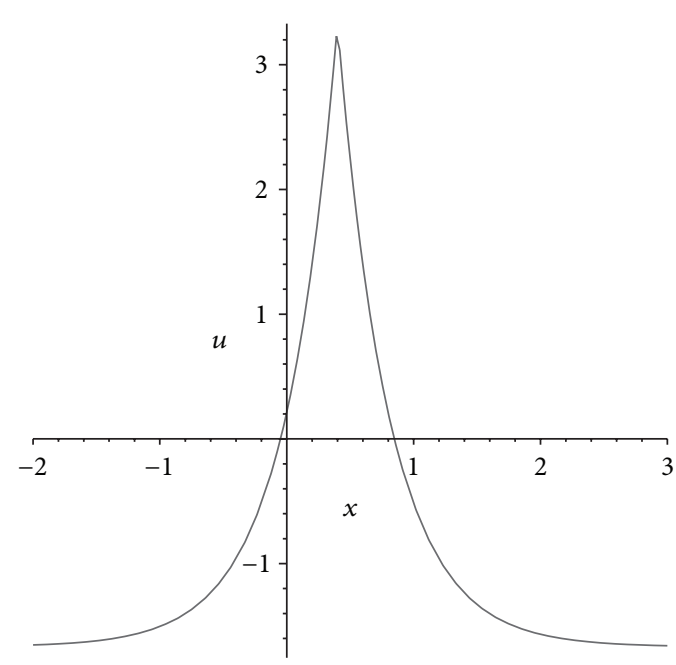

(a) Peakon wave defined by (51)

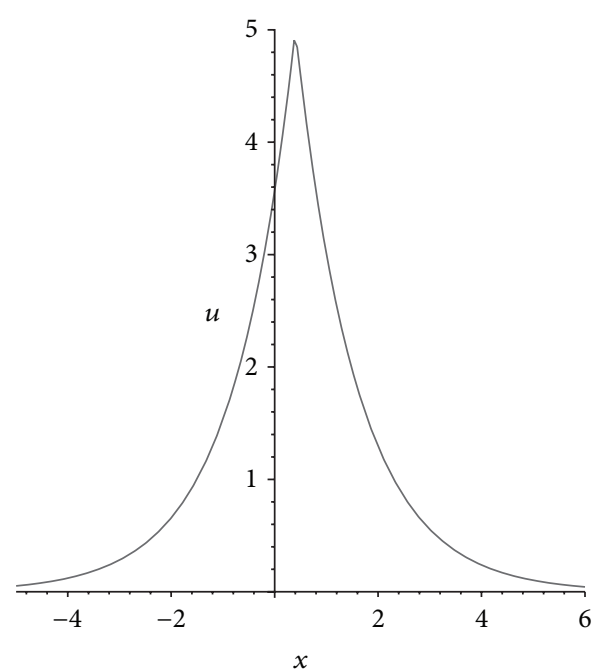

(b) Peakon wave defined by (57)

FIGURE 2: The graphs of profiles for the nonsmooth peakon solutions defined by (51) and (57) under different parametric values: (a) $v=1.4$; (b) $v=-1.4$.

where $\nu<0<\mu$. Thus, when the constants $c, g$, and $h$ satisfy the above conditions, (1) has a peakon solution as follows:

$$
u=-\frac{1}{6 \alpha \mu}+r \exp \left(-\sqrt{\frac{\sqrt{\nu^{2}-\mu \nu}-\nu}{\mu \nu}}|x-c t|\right)
$$

where $r$ is an arbitrary nonzero constant and $c$ is given above.

(b)When $g=h=0$ and $\beta$ can be regarded as a free parameter, we suppose that (8) has a peakon solution as the following form:

$$
u=\tilde{r} e^{-\widetilde{\delta}|\xi|},
$$

where the parameters $\widetilde{r}, \widetilde{\delta}$ can be determined further in the below discussions.

When $\xi \neq 0$, substituting (52) (i.e., $u=\tilde{r} e^{-\tilde{\delta} \sqrt{\xi^{2}}}$ ) into (8) we obtain

$$
\begin{gathered}
A \exp \left(-2 \delta \sqrt{\xi^{2}}\right)+B \exp \left(-3 \delta \sqrt{\xi^{2}}\right) \\
+C \exp \left(-4 \delta \sqrt{\xi^{2}}\right)=0
\end{gathered}
$$

where coefficients $A, B$, and $C$ satisfy

$$
\begin{gathered}
A=\frac{2(1-c) \widetilde{r}^{2}}{\nu}+\frac{2(\beta-c \nu) \widetilde{r}^{2} \widetilde{\delta}^{2}}{\nu}, \\
B=\frac{2 \alpha \widetilde{r}^{3}}{3 \nu}+\frac{2}{3} \alpha \widetilde{r}^{3} \widetilde{\delta}^{2}, \\
C=\frac{\mu \alpha^{2} \widetilde{r}^{4}}{\nu}+2 \mu \alpha^{2} \widetilde{r}^{4} \widetilde{\delta}^{2}+\mu^{2} \alpha^{2} \widetilde{r}^{4} \widetilde{\delta}^{4} .
\end{gathered}
$$

Let the coefficients of every terms of exp-function in (53) as zero; it follows

$$
A=0, \quad B=0, \quad C=0 .
$$

Solving the above group of equations yields

$$
\beta=\mu=\nu<0, \quad \tilde{\delta}=\frac{1}{\sqrt{-v}}, \quad \tilde{r}=\tilde{r}, \quad c=c .
$$

Thus, when the constants $g=h=0$ and $\beta=\mu=\nu, \widetilde{\delta}=$ $1 / \sqrt{-v}$, (1) has a peakon solution as follows:

$$
u=\widetilde{r} \exp \left(-\frac{1}{\sqrt{-v}}|x-c t|\right),
$$

where $\tilde{r}$ and $c$ are arbitrary nonzero constants.

In order to show the dynamical profiles of peakon solutions (51) and (57), we plot the graphs of them for $\alpha=$ $0.5, \beta=0.8, \mu=0.2, r=5, c=4, t=0.1$, which are shown in Figures 2(a) and 2(b).

\section{Different Kinds of Exact Solutions under the Special Case $v=0$}

Under the special case of parameter $v=0,(7)$ can be rewritten as

$$
-h-g u+\frac{1}{2}(1-c) u^{2}+\frac{1}{6} \alpha u^{3}+\frac{1}{4} \mu \alpha^{2} u^{4}+\frac{1}{2} \beta\left(\frac{d u}{d \xi}\right)^{2}=0 .
$$

Solving (58) in different kinds of parametric conditions, we obtain different kinds of exact traveling wave solutions including solitary wave solutions and kink wave solutions; see the below discussions. 
4.1. Different Kinds of Exact Traveling Wave Solutions for the Constants $g=h=0$. When two integral constants $g, h$ are both zero (i.e., $g=h=0$ ), (58) can be reduced to

$$
\left(\frac{d u}{d \xi}\right)^{2}=\frac{c-1}{\beta} u^{2}-\frac{\alpha}{3 \beta} u^{3}-\frac{\mu \alpha^{2}}{2 \beta} u^{4} .
$$

Under different parametric conditions solving (59), we obtain a series of exact traveling wave solutions as follows:

$$
u=\frac{6(c-1) \operatorname{sech}^{2}((1 / 2) \sqrt{(c-1) / \beta} \xi)}{2 \alpha+9 \alpha \mu(c-1)[1 \pm \tanh ((1 / 2) \sqrt{(c-1) / \beta} \xi)]^{2}}
$$

where $c>1, \beta>0$, and $\xi=x-c t$ :

$$
u=\frac{6(c-1)}{\alpha[1-\sqrt{18 \mu(1-c)-1} \sinh (\sqrt{(c-1) / \beta} \xi)]},
$$

where $c>1, \beta>0, \mu<-1 / 18(c-1)$, and $\xi=x-c t$ :

$$
u=\frac{6(c-1)}{\alpha[1+\sqrt{18 \mu(c-1)+1} \sin (\sqrt{(1-c) / \beta} \xi)]},
$$

where $c<1, \beta>0, \mu<-1 / 18(c-1)$, and $\xi=x-c t$ :

$$
u=\frac{6(c-1)}{\alpha[1-\sqrt{18 \mu(c-1)+1} \cosh (\sqrt{(c-1) / \beta} \xi)]},
$$

where $c>1, \beta>0, \mu>-1 / 18(c-1)$, and $\xi=x-c t$ :

$$
u=\frac{6(c-1)}{\alpha[1-\sqrt{18 \mu(c-1)+1} \cos (\sqrt{(1-c) / \beta} \xi)]},
$$

where $c<1, \beta>0, \mu>-1 / 18(c-1)$, and $\xi=x-c t$ :

$$
\begin{aligned}
u= & 3(c-1) \\
\times & \times\left(\alpha \left[\cosh ^{2}\left(\frac{1}{2} \sqrt{\frac{c-1}{\beta}} \xi\right)\right.\right. \\
& \left.\left.-3 \sqrt{\frac{\mu(1-c)}{2}} \sinh \left(\sqrt{\frac{c-1}{\beta}} \xi\right)\right]\right)^{-1},
\end{aligned}
$$

where $c>1, \beta>0, \mu<0$, and $\xi=x-c t$ :

$$
\begin{aligned}
& u=3(c-1) \\
& \times\left(\alpha \left[\cos ^{2}\left(\frac{1}{2} \sqrt{\frac{c-1}{\beta}} \xi\right)\right.\right. \\
& \left.\left.+3 \sqrt{\frac{\mu(c-1)}{2}} \sin \left(\sqrt{\frac{1-c}{\beta}} \xi\right)\right]\right)^{-1},
\end{aligned}
$$

where $c<1, \beta>0, \mu<0$, and $\xi=x-c t$ :

$$
u=-\frac{1}{6 \alpha \mu}\left[1 \pm \tanh \left(\frac{1}{12} \sqrt{-\frac{2}{\beta \mu} \xi}\right)\right]
$$

or

$$
u=-\frac{1}{6 \alpha \mu}\left[1 \pm \operatorname{coth}\left(\frac{1}{12} \sqrt{-\frac{2}{\beta \mu} \xi}\right)\right]
$$

where $\beta>0, \mu<0$, and $\xi=x-(1-(1 / 18 \mu)) t$.

Among these exact travelling wave solutions obtained in Section 4.1, it is worth pointing that the solutions (60) and (63) describe smooth solitary waves and the solution (67) describes kink wave and antikink wave (Figure 4). In order to show the dynamic profiles of solutions (60) and (63), we plot their graphs for $\alpha=0.5, \beta=0.8, c=5$, and $t=0.1$, which are shown in Figures 3(a) and 3(b).

In order to show the dynamic profiles of solution (67), we plot its graphs for $\alpha=0.5, \beta=0.8, \mu=-0.2$, and $t=0.1$, which are shown in Figures 3(a) and 3(b).

4.2. Exact Traveling Wave Solutions for the Constants $g \neq 0$ and $h=0$. When $g \neq 0$ and $h=0$, (58) can be reduced to

$$
-g u+\frac{1}{2}(1-c) u^{2}+\frac{1}{6} \alpha u^{3}+\frac{1}{4} \mu \alpha^{2} u^{4}+\frac{1}{2} \beta\left(\frac{d u}{d \xi}\right)^{2}=0 .
$$

If $\beta \mu<0$, then (69) can be rewritten as

$$
\begin{aligned}
& \frac{d u}{\sqrt{u\left[u^{3}+(2 / 3 \alpha \mu) u^{2}-\left(2(c-1) / \mu \alpha^{2}\right) u-\left(4 g / \mu \alpha^{2}\right)\right]}} \\
& = \pm \alpha \sqrt{-\frac{\mu}{2 \beta}} d \xi .
\end{aligned}
$$

By using factoring method, (70) can be rewritten in the following two forms:

$$
\begin{array}{r}
\frac{d u}{\sqrt{(u-0)\left(u-z_{0}\right)\left(u-z_{1}\right)\left(u-\overline{z_{1}}\right)}}= \pm \alpha \sqrt{-\frac{\mu}{2 \beta}} d \xi, \\
\left(z_{0}<0\right)
\end{array}
$$

or

$$
\begin{array}{r}
\frac{d u}{\sqrt{\left(u-z_{0}\right)(u-0)\left(u-z_{1}\right)\left(u-\overline{z_{1}}\right)}}= \pm \alpha \sqrt{-\frac{\mu}{2 \beta}} d \xi, \\
\left(z_{0}>0\right),
\end{array}
$$

where $z_{0}, z_{1}$, and $\overline{z_{1}}$ are roots of the equation $u^{3}+(2 / 3 \alpha \mu) u^{2}-$ $\left(2(c-1) / \mu \alpha^{2}\right) u-\left(4 g / \mu \alpha^{2}\right)=0$ and the $z_{0}$ is real root; the $z_{1}, \overline{z_{1}}$ are conjugate complex roots. $z_{0}, z_{1}$, and $\overline{z_{1}}$ can be expressed by the parameters $\alpha, \mu, c$, and $g$, and here we 


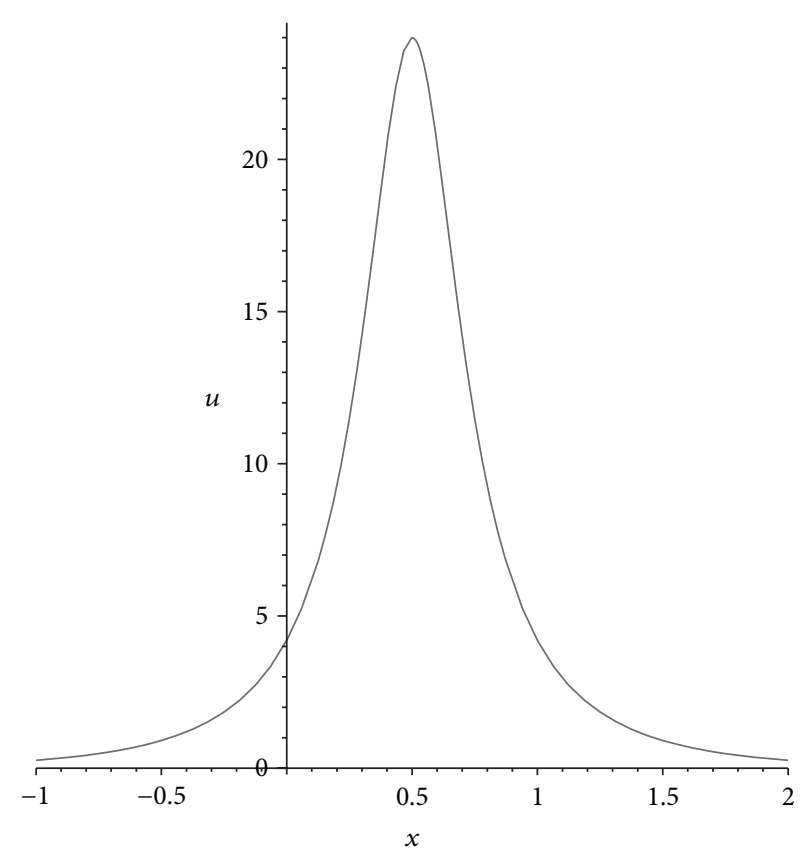

(a) Bright solitary wave defined by (60)

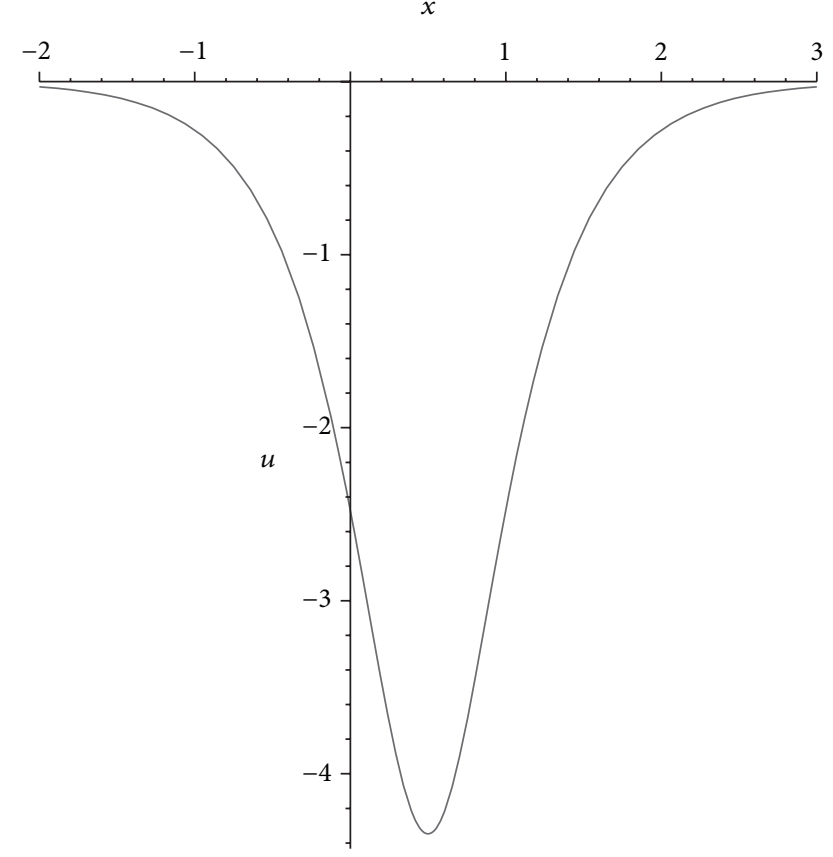

(b) Dark solitary wave defined by (63)

FIGURE 3: The graphs of profiles for smooth solitary wave solutions defined by (60) and (63) under different parametric values: (a) $\mu=0.7$; (b) $\mu=2$.

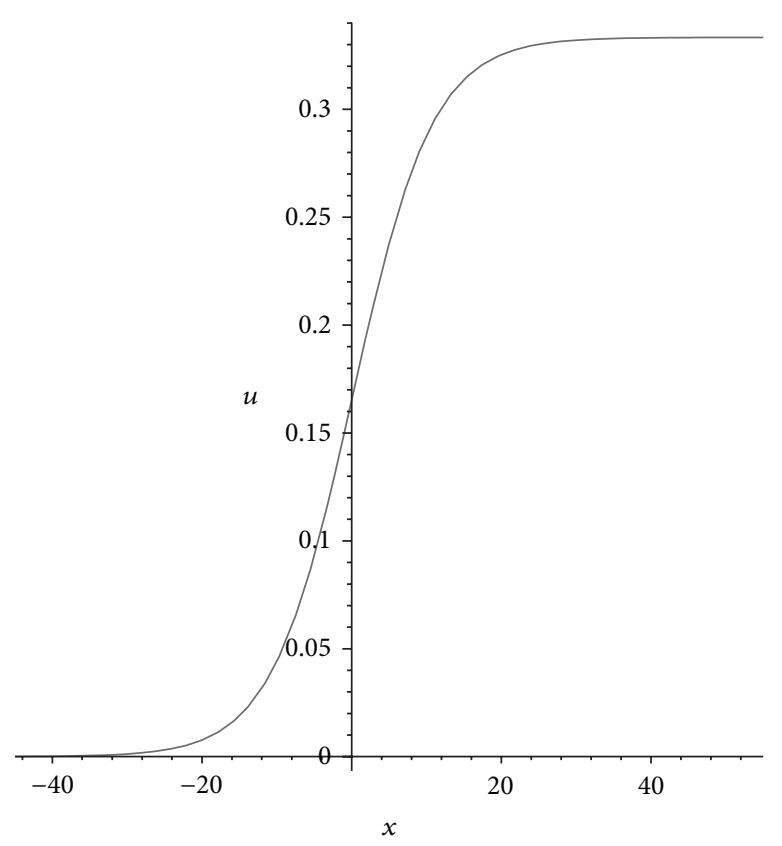

(a) Kink wave

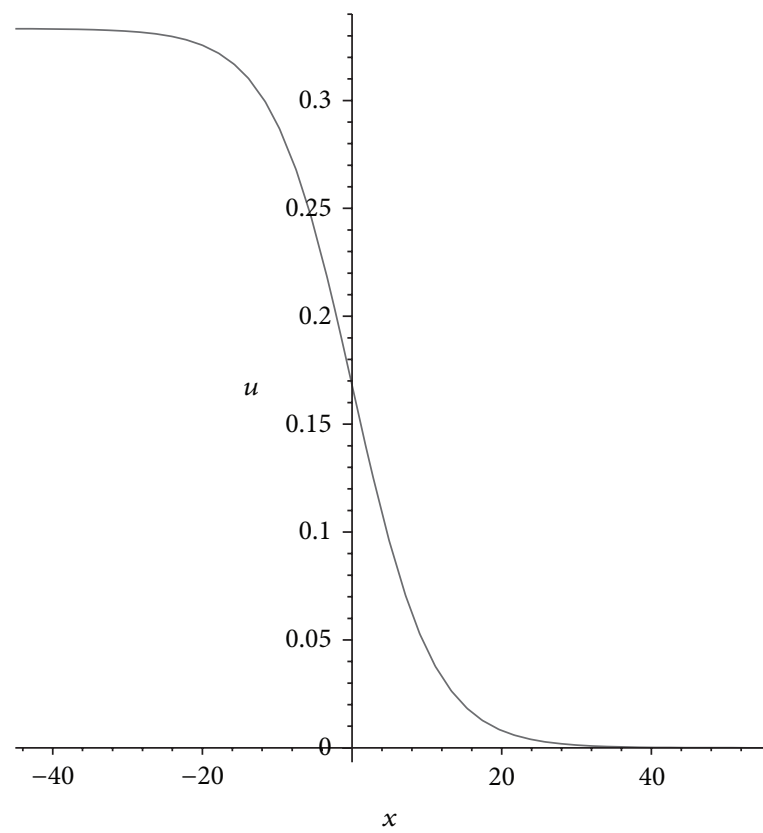

(b) Antikink wave

FIgURE 4: The graphs of profiles for kink wave and antikink wave solutions defined by (67) with “+” and “-”.

omit their expressions because they are very complex. But we can always obtain their values by using computer once the parameters $\alpha, \mu, c$, and $g$ are fixed on concrete values. For example, taking $\alpha=0.8, c=5, \mu=-5, g=-4$, we get $z_{0}=$
$1.204421920, z_{1}=-0.6855442934+1.918696998 i$, and $\overline{z_{1}}=$ $-0.6855442934-1.918696998 i$; taking $\alpha=0.8, c=5, \mu=$ $-5, g=4$, we get $z_{0}=-1.276547333, z_{1}=0.5549403329+$ $1.899699037 i$, and $\overline{z_{1}}=0.5549403329+1.899699037 i$. 
Solving (71) and (72), we obtain two periodic wave solutions of (1) as follows:

$$
\begin{aligned}
u= & z_{0}\left|z_{1}\right|\left[\operatorname{cn}\left(\widetilde{\omega}_{1} \xi, m_{1}\right) \mp 1\right] \\
& \times\left(\left|z_{1}\right|\left[\operatorname{cn}\left(\widetilde{\omega}_{1} \xi, m_{1}\right) \mp 1\right]\right. \\
& \left.+\sqrt{z_{0}^{2}-2 z_{0} \cdot \operatorname{Re}\left(z_{1}\right)+\left|z_{1}\right|^{2}}\left[\operatorname{cn}\left(\widetilde{\omega}_{1} \xi, m_{1}\right) \pm 1\right]\right)^{-1},
\end{aligned}
$$

where

$$
\widetilde{\omega}_{1}=\alpha \sqrt{\frac{-\mu\left|z_{1}\right| \sqrt{z_{0}^{2}-2 z_{0} \cdot \operatorname{Re}\left(z_{1}\right)+\left|z_{1}\right|^{2}}}{2 \beta}}
$$

$m_{1}$

$$
=\frac{1}{2} \sqrt{\frac{2\left|z_{1}\right|^{2}-2 z_{0} \cdot \operatorname{Re}\left(z_{1}\right)+2\left|z_{1}\right| \sqrt{z_{0}^{2}-2 z_{0} \cdot \operatorname{Re}\left(z_{1}\right)+\left|z_{1}\right|^{2}}}{\left|z_{1}\right| \sqrt{z_{0}^{2}-2 z_{0} \cdot \operatorname{Re}\left(z_{1}\right)+\left|z_{1}\right|^{2}}}} .
$$

If $\beta \mu>0$, then (69) can be rewritten as

$$
\begin{aligned}
& \frac{d u}{\left.\sqrt{-u\left[u^{3}+(2 / 3 \alpha \mu) u^{2}-\left(2(c-1) / \mu \alpha^{2}\right) u-\left(4 g / \mu \alpha^{2}\right)\right.}\right]} \\
& = \pm \alpha \sqrt{\frac{\mu}{2 \beta}} d \xi .
\end{aligned}
$$

By using factoring method, (75) can be rewritten in the following two forms:

$$
\frac{d u}{\sqrt{(0-u)\left(u-z_{0}\right)\left(u-z_{1}\right)\left(u-\overline{z_{1}}\right)}}= \pm \alpha \sqrt{\frac{\mu}{2 \beta}} d \xi,
$$

$$
\left(z_{0}<0\right)
$$

or

$$
\begin{array}{r}
\frac{d u}{\sqrt{\left(z_{0}-u\right)(u-0)\left(u-z_{1}\right)\left(u-\overline{z_{1}}\right)}}= \pm \alpha \sqrt{\frac{\mu}{2 \beta}} d \xi, \\
\left(z_{0}>0\right),
\end{array}
$$

where $z_{0}, z_{1}$, and $\overline{z_{1}}$ are same as the above case.

Solving (76) and (77), we obtain two periodic wave solutions of (1) as follows:

$$
\begin{aligned}
& u=z_{0}\left|z_{1}\right|\left[1+\operatorname{cn}\left(\widetilde{\omega}_{2} \xi, m_{2}\right)\right] \\
& \quad \times\left(\left|z_{1}\right|\left[1+\operatorname{cn}\left(\widetilde{\omega}_{2} \xi, m_{2}\right)\right]\right. \\
& \left.\quad+\sqrt{z_{0}^{2}-2 z_{0} \cdot \operatorname{Re}\left(z_{1}\right)+\left|z_{1}\right|^{2}}\left[1-\operatorname{cn}\left(\widetilde{\omega}_{2} \xi, m_{2}\right)\right]\right)^{-1},
\end{aligned}
$$

$$
\begin{aligned}
u= & z_{0} \sqrt{z_{0}^{2}-2 z_{0} \cdot \operatorname{Re}\left(z_{1}\right)+\left|z_{1}\right|^{2}}\left[1+\operatorname{cn}\left(\widetilde{\omega}_{2} \xi, m_{2}\right)\right] \\
& \times\left(\left|z_{1}\right|\left[1-\operatorname{cn}\left(\widetilde{\omega}_{2} \xi, m_{2}\right)\right]\right. \\
& +\sqrt{z_{0}^{2}-2 z_{0} \cdot \operatorname{Re}\left(z_{1}\right)+\left|z_{1}\right|^{2}} \\
& \left.\times\left[1+\operatorname{cn}\left(\widetilde{\omega}_{2} \xi, m_{2}\right)\right]\right)^{-1},
\end{aligned}
$$

where

$$
\widetilde{\omega}_{2}=\alpha \sqrt{\frac{\mu\left|z_{1}\right| \sqrt{z_{0}^{2}-2 z_{0} \cdot \operatorname{Re}\left(z_{1}\right)+\left|z_{1}\right|^{2}}}{2 \beta}}
$$

$$
=\frac{1}{2} \sqrt{\frac{2 z_{0} \cdot \operatorname{Re}\left(z_{1}\right)-2\left|z_{1}\right|^{2}+2\left|z_{1}\right| \sqrt{z_{0}^{2}-2 z_{0} \cdot \operatorname{Re}\left(z_{1}\right)+\left|z_{1}\right|^{2}}}{\left|z_{1}\right| \sqrt{z_{0}^{2}-2 z_{0} \cdot \operatorname{Re}\left(z_{1}\right)+\left|z_{1}\right|^{2}}}} .
$$

4.3. Exact Traveling Wave Solutions for the Constants $g \neq 0$ and $h \neq 0$. When $g \neq 0$ and $h \neq 0$, (58) can be rewritten as

$$
\begin{aligned}
d u\left(-\frac{\mu \alpha^{2}}{2 \beta}\left[u^{4}+\frac{2}{3 \mu \alpha} u^{3}-\frac{2(c-1)}{\mu \alpha^{2}} u^{2}\right.\right. \\
\left.\left.\quad-\frac{4 g}{\mu \alpha^{2}} u-\frac{4 h}{\mu \alpha^{2}}\right]\right)^{-1 / 2}= \pm d \xi .
\end{aligned}
$$

The types of solutions of (81) contain many cases due to the roots of the quartic equation $u^{4}+(2 / 3 \mu \alpha) u^{3}-(2(c-$ 1) $\left./ \mu \alpha^{2}\right) u^{2}-\left(4 g / \mu \alpha^{2}\right) u-\left(4 h / \mu \alpha^{2}\right)=0$ which is in $(81)$ and have many possibilities, but all solutions of (1) under different kinds of cases are periodic solutions of Jacobian elliptic function type such as the forms of solutions (73), (78), and (79). For convenience to discuss, here we only consider one case which this quartic equation has four real roots. Obviously, we can always obtain the real roots of this quartic equation by using computer once the parameters $\alpha, \beta, \mu, c, g$, and $h$ are fixed on concrete values. Supposing $\phi_{1}, \phi_{2}, \phi_{3}$, and $\phi_{4}$ are four real roots of this quartic equation, we will obtain different kinds of periodic wave solutions of Jacobian elliptic function type for (1); see the following discussions.

Case 1. Under $\beta \mu<0$, respectively, taking different root of the $\phi_{1}, \phi_{2}, \phi_{3}$, and $\phi_{4}$ as initial value to integrate (81) yields

$$
\int_{\phi_{1}}^{u} \frac{d \widetilde{u}}{\sqrt{\left(\tilde{u}-\phi_{1}\right)\left(\tilde{u}-\phi_{2}\right)\left(\tilde{u}-\phi_{3}\right)\left(\tilde{u}-\phi_{4}\right)}}= \pm \alpha \sqrt{-\frac{\mu}{2 \beta}} \int_{0}^{\xi} d \tilde{\xi},
$$

where $u>\phi_{1}>\phi_{2}>\phi_{3}>\phi_{4}$ and

$$
\int_{u}^{\phi_{2}} \frac{d \tilde{u}}{\sqrt{\left(\phi_{1}-\tilde{u}\right)\left(\phi_{2}-\tilde{u}\right)\left(\tilde{u}-\phi_{3}\right)\left(\tilde{u}-\phi_{4}\right)}}= \pm \alpha \sqrt{-\frac{\mu}{2 \beta}} \int_{0}^{\xi} d \tilde{\xi},
$$


where $\phi_{1}>\phi_{2}>u \geq \phi_{3}>\phi_{4}$ and

$$
\int_{\phi_{3}}^{u} \frac{d \tilde{u}}{\sqrt{\left(\phi_{1}-\tilde{u}\right)\left(\phi_{2}-\tilde{u}\right)\left(\tilde{u}-\phi_{3}\right)\left(\tilde{u}-\phi_{4}\right)}}= \pm \alpha \sqrt{-\frac{\mu}{2 \beta}} \int_{0}^{\xi} d \tilde{\xi},
$$

where $\phi_{1}>\phi_{2} \geq u>\phi_{3}>\phi_{4}$ and

$$
\int_{u}^{\phi_{4}} \frac{d \tilde{u}}{\sqrt{\left(\phi_{1}-\tilde{u}\right)\left(\phi_{2}-\tilde{u}\right)\left(\phi_{3}-\tilde{u}\right)\left(\phi_{4}-\tilde{u}\right)}}= \pm \alpha \sqrt{-\frac{\mu}{2 \beta}} \int_{0}^{\xi} d \tilde{\xi},
$$

where $\phi_{1}>\phi_{2}>u>\phi_{3}>\phi_{4}>u$.

Respectively, completing the above integrals in (82), (83), (84), and (85), we obtain four periodic wave solutions of Jacobian elliptic function type of (1) as follows:

$$
\begin{aligned}
& u=\frac{\left(\phi_{1} \phi_{2}-\phi_{2} \phi_{4}\right) \operatorname{sn}^{2}\left(\Omega_{1} \xi, k_{1}\right)+\left(\phi_{1} \phi_{4}-\phi_{1} \phi_{2}\right)}{\left(\phi_{1}-\phi_{4}\right) \operatorname{sn}^{2}\left(\Omega_{1} \xi, k_{1}\right)+\left(\phi_{4}-\phi_{2}\right)}, \\
& u=\frac{\left(\phi_{1} \phi_{2}-\phi_{1} \phi_{3}\right) \operatorname{sn}^{2}\left(\Omega_{1} \xi, k_{1}\right)+\left(\phi_{2} \phi_{3}-\phi_{1} \phi_{2}\right)}{\left(\phi_{2}-\phi_{3}\right) \operatorname{sn}^{2}\left(\Omega_{1} \xi, k_{1}\right)+\left(\phi_{3}-\phi_{1}\right)}, \\
& u=\frac{\left(\phi_{1} \phi_{2}-\phi_{2} \phi_{4}\right) \operatorname{sn}^{2}\left(\Omega_{1} \xi, k_{1}\right)+\left(\phi_{1} \phi_{4}-\phi_{1} \phi_{2}\right)}{\left(\phi_{1}-\phi_{4}\right) \operatorname{sn}^{2}\left(\Omega_{1} \xi, k_{1}\right)+\left(\phi_{4}-\phi_{2}\right)}, \\
& u=\frac{\left(\phi_{2} \phi_{4}-\phi_{3} \phi_{4}\right) \operatorname{sn}^{2}\left(\Omega_{1} \xi, k_{1}\right)+\left(\phi_{3} \phi_{4}-\phi_{2} \phi_{3}\right)}{\left(\phi_{2}-\phi_{3}\right) \operatorname{sn}^{2}\left(\Omega_{1} \xi, k_{1}\right)+\left(\phi_{4}-\phi_{2}\right)}, \\
& u=\frac{\left(\phi_{1} \phi_{2}-\phi_{2} \phi_{4}\right) \operatorname{sn}^{2}\left(\Omega_{1} \xi, k_{1}\right)+\left(\phi_{1} \phi_{4}-\phi_{1} \phi_{2}\right)}{\left(\phi_{1}-\phi_{4}\right) \operatorname{sn}^{2}\left(\Omega_{1} \xi, k_{1}\right)+\left(\phi_{4}-\phi_{2}\right)}, \\
& u=\frac{\left(\phi_{1} \phi_{3}-\phi_{3} \phi_{4}\right) \operatorname{sn}^{2}\left(\Omega_{1} \xi, k_{1}\right)+\left(\phi_{3} \phi_{4}-\phi_{1} \phi_{4}\right)}{\left(\phi_{1}-\phi_{4}\right) \operatorname{sn}^{2}\left(\Omega_{1} \xi, k_{1}\right)+\left(\phi_{3}-\phi_{1}\right)},
\end{aligned}
$$

where $\Omega_{1}= \pm(\alpha / 2) \sqrt{-\mu\left(\phi_{1}-\phi_{3}\right)\left(\phi_{2}-\phi_{4}\right) / 2 \beta}$ and $k_{1}=$ $\sqrt{\left(\phi_{2}-\phi_{3}\right)\left(\phi_{1}-\phi_{4}\right) /\left(\phi_{1}-\phi_{3}\right)\left(\phi_{2}-\phi_{4}\right)}$.

Case 2. Under $\beta \mu>0$, respectively, taking different root of the $\phi_{1}, \phi_{2}, \phi_{3}$, and $\phi_{4}$ as initial value to integrate (81) yields

$$
\int_{u}^{\phi_{1}} \frac{d \tilde{u}}{\sqrt{\left(\phi_{1}-\tilde{u}\right)\left(\tilde{u}-\phi_{2}\right)\left(\tilde{u}-\phi_{3}\right)\left(\tilde{u}-\phi_{4}\right)}}= \pm \alpha \sqrt{\frac{\mu}{2 \beta}} \int_{0}^{\xi} d \tilde{\xi},
$$

where $\phi_{1}>u \geq \phi_{2}>\phi_{3}>\phi_{4}$ and

$$
\int_{\phi_{2}}^{u} \frac{d \tilde{u}}{\sqrt{\left(\phi_{1}-\tilde{u}\right)\left(\tilde{u}-\phi_{2}\right)\left(\tilde{u}-\phi_{3}\right)\left(\tilde{u}-\phi_{4}\right)}}= \pm \alpha \sqrt{\frac{\mu}{2 \beta}} \int_{0}^{\xi} d \tilde{\xi}
$$

where $\phi_{1} \geq u>\phi_{2}>\phi_{3}>\phi_{4}$ and

$$
\int_{u}^{\phi_{3}} \frac{d \tilde{u}}{\sqrt{\left(\phi_{1}-\tilde{u}\right)\left(\phi_{2}-\tilde{u}\right)\left(\phi_{3}-\tilde{u}\right)\left(\tilde{u}-\phi_{4}\right)}}= \pm \alpha \sqrt{\frac{\mu}{2 \beta}} \int_{0}^{\xi} d \tilde{\xi},
$$

where $\phi_{1}>\phi_{2}>\phi_{3}>u \geq \phi_{4}$ and

$$
\int_{\phi_{4}}^{u} \frac{d \tilde{u}}{\sqrt{\left(\phi_{1}-\tilde{u}\right)\left(\phi_{2}-\tilde{u}\right)\left(\phi_{3}-\tilde{u}\right)\left(\tilde{u}-\phi_{4}\right)}}= \pm \alpha \sqrt{\frac{\mu}{2 \beta}} \int_{0}^{\xi} d \tilde{\xi},
$$

where $\phi_{1}>\phi_{2}>\phi_{3} \geq u>\phi_{4}$.

Similarly completing the above integrals in (87), (88), (89), and (90), we obtain another four periodic wave solutions of Jacobian elliptic function type of (1) as follows:

$$
\begin{aligned}
& u=\frac{\left(\phi_{1} \phi_{4}-\phi_{2} \phi_{4}\right) \operatorname{sn}^{2}\left(\Omega_{2} \xi, k_{2}\right)+\left(\phi_{1} \phi_{2}-\phi_{1} \phi_{4}\right)}{\left(\phi_{1}-\phi_{2}\right) \operatorname{sn}^{2}\left(\Omega_{2} \xi, k_{2}\right)+\left(\phi_{2}-\phi_{4}\right)}, \\
& u=\frac{\left(\phi_{1} \phi_{3}-\phi_{2} \phi_{3}\right) \operatorname{sn}^{2}\left(\Omega_{2} \xi, k_{2}\right)+\left(\phi_{2} \phi_{3}-\phi_{1} \phi_{2}\right)}{\left(\phi_{1}-\phi_{2}\right) \operatorname{sn}^{2}\left(\Omega_{2} \xi, k_{2}\right)+\left(\phi_{3}-\phi_{1}\right)}, \\
& u=\frac{\left(\phi_{2} \phi_{3}-\phi_{2} \phi_{4}\right) \operatorname{sn}^{2}\left(\Omega_{2} \xi, k_{2}\right)+\left(\phi_{3} \phi_{4}-\phi_{2} \phi_{3}\right)}{\left(\phi_{3}-\phi_{4}\right) \operatorname{sn}^{2}\left(\Omega_{2} \xi, k_{2}\right)+\left(\phi_{4}-\phi_{2}\right)}, \\
& u=\frac{\left(\phi_{1} \phi_{3}-\phi_{1} \phi_{4}\right) \operatorname{sn}^{2}\left(\Omega_{2} \xi, k_{2}\right)+\left(\phi_{1} \phi_{4}-\phi_{3} \phi_{4}\right)}{\left(\phi_{3}-\phi_{4}\right) \operatorname{sn}^{2}\left(\Omega_{2} \xi, k_{2}\right)+\left(\phi_{3}-\phi_{1}\right)},
\end{aligned}
$$

where $\Omega_{2}= \pm(\alpha / 2) \sqrt{\mu\left(\phi_{1}-\phi_{3}\right)\left(\phi_{2}-\phi_{4}\right) / 2 \beta}$ and $k_{2}=$ $\sqrt{\left(\phi_{1}-\phi_{2}\right)\left(\phi_{3}-\phi_{4}\right) /\left(\phi_{1}-\phi_{3}\right)\left(\phi_{2}-\phi_{4}\right)}$.

In Sections 4.2 and 4.3, all the exact solutions obtained by us are periodic solutions of Jacobian elliptic function types. As an example, we plot the graphs of profiles of the solutions (78) and (79) for $\alpha=0.3, \beta=0.8, c=1, \mu=2$, and $t=0.1$, which are shown in Figures 5(a) and 5(b).

\section{Conclusions}

Though Frobenius' idea is a well-known general method, it can solve some very complex PDE models with highly nonlinear terms and high order terms such as (1) when it combines with the integral bifurcation method. In this work, by using Frobenius' idea together with integral bifurcation method, we study the third order nonlinear water wave model (1). Under different kinds of parametric conditions, we obtain eight types of exact travelling wave solutions including the smooth solitary wave solutions (60), (63), and (65), the nonsmooth peakon wave solutions (51) and (57), the kink wave and antikink wave solutions (67) and (68), the smooth periodic wave solutions of trigonometric function type (14), (16), (21), (24), (28), (31), (34), (36), (38), (40), and (43), the nonsmooth periodic wave solutions of trigonometric function type (62), (64), and (66), the periodic wave solutions of Jacobian elliptic function type (78), (86), (91), and (92), the hyperbolic function solutions (13), (15), (20), (23), (27), (30), (33), (35), (44), and (61), and the rational function solution (45). Though the types of these solutions obtained in this work are not new and they are familiar types, the results of (1) obtained by us in this paper did not appear in any existing literatures. Particularly, compared with reference [45], all results obtained in this paper are new. Among these solutions obtained in this paper, some of them have direct 


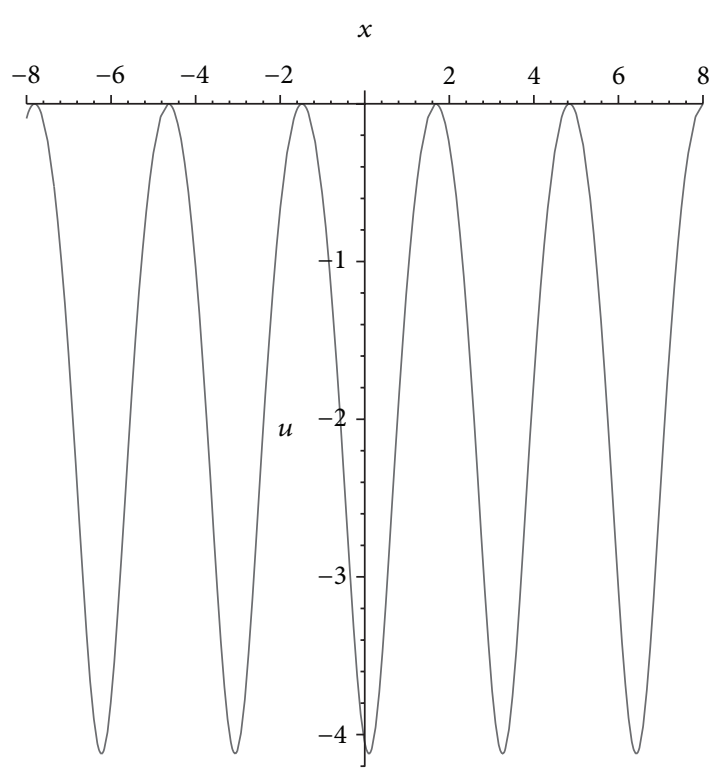

(a) Periodic wave defined by $(78)$

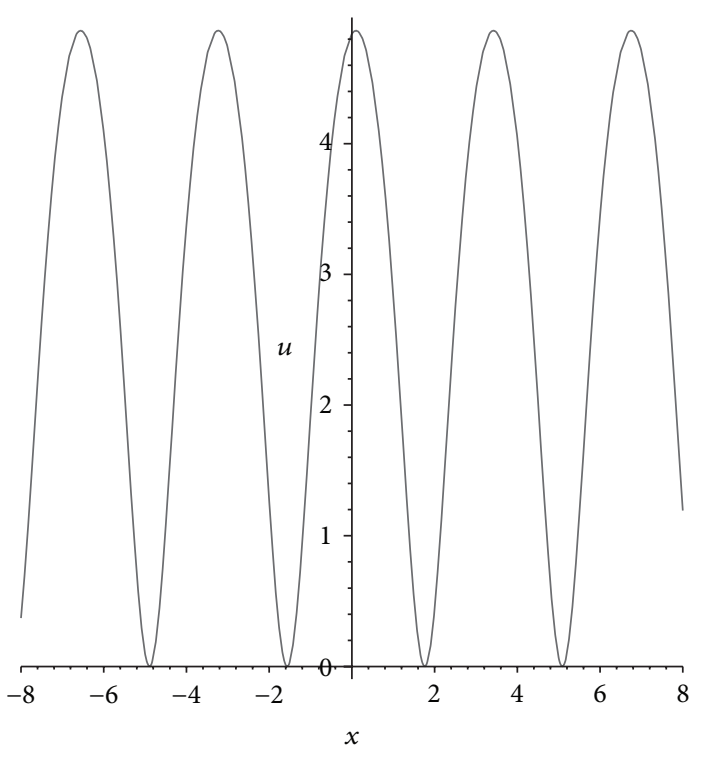

(b) Periodic wave defined by (79)

Figure 5: The graphs of profiles for periodic wave solutions of Jacobin elliptic function types defined by (78) and (79) under different parametric values: (a) $g=4$; (b) $g=-4$.

physical applications. For example, using the smooth solitary wave solutions, nonsmooth peakon wave solutions, and kink and antikink wave solutions, we can explain lots of motion phenomena for water wave; indeed (1) is just a very important water wave model.

\section{Conflict of Interests}

The author declares that there is no conflict of interests regarding the publication of this paper.

\section{Acknowledgments}

This work is supported by the Natural Science Foundation of China under Grant no. 11361023, the Natural Science Foundation of Scientific and Technical Committee of Chongqing City under Grant no. cstc2014jcyjA00014, the Natural Science Foundation of Chongqing Normal University under Grant no. 13XLR20, and the Program Foundation of Chongqing Innovation Team Project in University under Grant no. KJTD201308.

\section{References}

[1] C. S. Gardner, J. M. Greene, M. D. Kruskal, and R. M. Miura, "Method for solving the Korteweg-deVries equation," Physical Review Letters, vol. 19, no. 19, pp. 1095-1097, 1967.

[2] C. S. Gardner, J. M. Greene, M. D . Kruskal, and R. M. Miura, "Korteweg-deVries equation and generalization. VI. Methods for exact solution," Communications on Pure and Applied Mathematics, vol. 27, pp. 97-133, 1974.

[3] V. B. Matveev and M. A. Salle, Darboux Transformations and Solitons, Springer, Berlin, Germany, 1991.
[4] C. H. Gu, B. L. Guo, Y. S. Li et al., Soliton Theory and Its Applications, Springer, New York, NY, USA, 1995.

[5] C. Rogers and W. K. Schief, Bäcklund and Darboux Transformations, Cambridge Texts in Applied Mathematics, Cambridge University Press, Cambridge, UK, 2002.

[6] M. R. Mirura, Bäcklund Transformation, Springer, Berlin, Germany, 1978.

[7] M. Wadati, H. Sanuki, and K. Konno, "Relationships among inverse method, Bäcklund transformation and an infinite number of conservation laws," Progress of Theoretical Physics, vol. 53, no. 2, pp. 419-436, 1975.

[8] I. T. Khabibullin, "The Bäcklund transformation and integrable initial boundary value problems," Mathematical Notes of the Academy of Sciences of the USSR, vol. 49, no. 4, pp. 418-423, 1991.

[9] R. Hirota, "Exact solution of the korteweg-de vries equation for multiple collisions of solitons," Physical Review Letters, vol. 27, no. 18, pp. 1192-1194, 1971.

[10] R. Hirota, The Direct Method in Soliton Theory, edited and translated by A. Nimmo, C. Gilson, Cambridge University Press, Cambridge, UK, 2004.

[11] G. W. Bluman and S. Kumei, Symmetries and Differential Equation, vol. 154 of Applied Mathematica Sciences, Springer, New York, NY, USA, 1989.

[12] P. J. Olver, Applications of Lie Groups to Differential Equations, Springer, New York, NY, USA, 1993.

[13] P. A. Clarkson and M. D. Kruskal, "New similarity reductions of the Boussinesq equation," Journal of Mathematical Physics, vol. 30, no. 10, pp. 2201-2213, 1989.

[14] P. A. Clarkson, "Nonclassical symmetry reductions of the Boussinesq equation," Chaos, Solitons and Fractals, vol. 5, no. 12, pp. 2261-2301, 1995

[15] P. A. Clarkson and E. L. Mansfield, "Symmetry reductions and exact solutions of shallow water wave equations," Acta Applicandae Mathematicae, vol. 39, no. 1-3, pp. 245-276, 1995. 
[16] W. Hereman, P. P. Banerjee, A. Korpel, G. Assanto, A. van Immerzeele, and A. Meerpoel, "Exact solitary wave solutions of nonlinear evolution and wave equations using a direct algebraic method," Journal of Physics A: Mathematical and General, vol. 19, no. 5, pp. 607-628, 1986.

[17] P. W. Doyle, "Separation of variables for scalar evolution equations in one space dimension," Journal of Physics A: Mathematical and General, vol. 29, no. 23, pp. 7581-7595, 1996.

[18] P. W. Doyle and P. J. Vassiliou, "Separation of variables for the 1-dimensional non-linear diffusion equation," International Journal of Non-Linear Mechanics, vol. 33, no. 2, pp. 315-326, 1998.

[19] J. Weiss, M. Tabor, and G. Carnevale, “The Painlevé property for partial differential equations," Journal of Mathematical Physics, vol. 24, no. 3, pp. 522-526, 1983.

[20] R. Conte, "Invariant painlevé analysis of partial differential equations," Physics Letters A, vol. 140, no. 7-8, pp. 383-390, 1989.

[21] W. Malfliet and W. Hereman, "The tanh method. I. Exact solutions of nonlinear evolution and wave equations," Physica Scripta, vol. 54, no. 6, pp. 563-568, 1996.

[22] A.-M. Wazwaz, "The tanh method for traveling wave solutions of nonlinear equations," Applied Mathematics and Computation, vol. 154, no. 3, pp. 713-723, 2004.

[23] A. M. Wazwaz, "A sine-cosine method for handling nonlinear wave equations," Mathematical and Computer Modelling, vol. 40, no. 5-6, pp. 499-508, 2004.

[24] A. Wazwaz, "The sine-cosine method for obtaining solutions with compact and noncompact structures," Applied Mathematics and Computation, vol. 159, no. 2, pp. 559-576, 2004.

[25] J. He and X. Wu, "Exp-function method for nonlinear wave equations," Chaos, Solitons and Fractals, vol. 30, no. 3, pp. 700708, 2006.

[26] W. X. Ma, T. Huang, and Y. Zhang, "A multiple exp-function method for nonlinear differential equations and its application," Physica Scripta, vol. 82, no. 6, Article ID 065003, 2010.

[27] J. Li and Z. Liu, "Smooth and non-smooth traveling waves in a nonlinearly dispersive equation," Applied Mathematical Modelling, vol. 25, no. 1, pp. 41-56, 2000.

[28] J. Li and Z. Liu, "Traveling wave solutions for a class of nonlinear dispersive equations," Chinese Annals of Mathematics, vol. 23, no. 3, pp. 397-418, 2002.

[29] Y. Zhou, M. Wang, and Y. Wang, "Periodic wave solutions to a coupled KdV equations with variable coefficients," Physics Letters A, vol. 308, no. 1, pp. 31-36, 2003.

[30] J. Liu and K. Yang, "The extended F-expansion method and exact solutions of nonlinear PDEs," Chaos, Solitons and Fractals, vol. 22, no. 1, pp. 111-121, 2004.

[31] M. Wang, X. Li, and J. Zhang, "The $\left(G^{\prime} / G\right)$-expansion method and travelling wave solutions of nonlinear evolution equations in mathematical physics," Physics Letters A, vol. 372, no. 4, pp. 417-423, 2008.

[32] E. M. E. Zayed and K. A. Gepreel, "The ( $\left.G^{\prime} / G\right)$-expansion method for finding traveling wave solutions of nonlinear partial differential equations in mathematical physics," Journal of Mathematical Physics, vol. 50, no. 1, Article ID 013502, 2009.

[33] W. Rui, B. He, Y. Long, and C. Chen, "The integral bifurcation method and its application for solving a family of thirdorder dispersive PDEs," Nonlinear Analysis: Theory, Methods \& Applications, vol. 69, no. 4, pp. 1256-1267, 2008.
[34] R. Weiguo, L. Yao, H. Bin, and L. Zhenyang, "Integral bifurcation method combined with computer for solving a higher order wave equation of KdV type," International Journal of Computer Mathematics, vol. 87, no. 1, pp. 119-128, 2010.

[35] W. Rui, B. He, S. Xie, and Y. Long, "Application of the integral bifurcation method for solving modified Camassa-Holm and Degasperis-Procesi equations," Nonlinear Analysis: Theory, Methods \& Applications, vol. 71, no. 7-8, pp. 3459-3470, 2009.

[36] W. Rui, “The integral bifurcation method combined with factoring technique for investigating exact solutions and their dynamical properties of a generalized Gardner equation," Nonlinear Dynamics, vol. 76, no. 2, pp. 1529-1542, 2014.

[37] W. X. Ma, H. Wu, and J. He, "Partial differential equations possessing Frobenius integrable decompositions," Physics Letters A, vol. 364, no. 1, pp. 29-32, 2007.

[38] W. X. Ma and J. H. Lee, "A transformed rational function method and exact solutions to the $3+1$ dimensional JimboMiwa equation," Chaos, Solitons \& Fractals, vol. 42, no. 3, pp. 1356-1363, 2009.

[39] W. X. Ma and B. Fuchssteiner, "Explicit and exact solutions to a Kolmogorov-Petrovskii-PISkunov equation," International Journal of Non-Linear Mechanics, vol. 31, no. 3, pp. 329-338, 1996.

[40] A. S. Fokas, "On a class of physically important integrable equations," Physica D: Nonlinear Phenomena, vol. 87, no. 1-4, pp. 145150, 1995.

[41] B. Fuchssteiner and A. S. Fokas, "Symplectic structures, their Bäcklund transformations and hereditary symmetries," Physica D, vol. 4, no. 1, pp. 47-66, 1981/82.

[42] B. Fuchssteiner, "The Lie algebra structure of nonlinear evolution equations admitting infinite-dimensional abelian symmetry groups," Progress of Theoretical Physics, vol. 65, no. 3, pp. 861876, 1981.

[43] H. Yang and W. Rui, "New Lax pairs and Darboux transformation and its application to a shallow water wave model of generalized KdV type," Mathematical Problems in Engineering, vol. 2013, Article ID 548690, 8 pages, 2013.

[44] Q. Bi, "Wave patterns associated with a singular line for a biHamiltonian system," Physics Letter A, vol. 369, no. 5-6, pp. 407417, 2007.

[45] J. Li and J. Zhang, "Bifurcations of travelling wave solutions for the generalization form of the modified KdV equation," Chaos, Solitons \& Fractals, vol. 21, no. 4, pp. 889-913, 2004. 


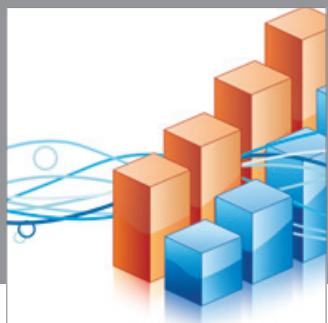

Advances in

Operations Research

mansans

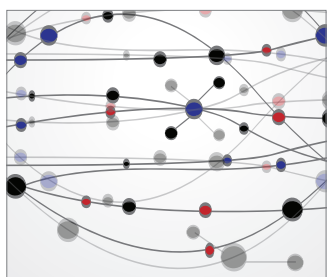

The Scientific World Journal
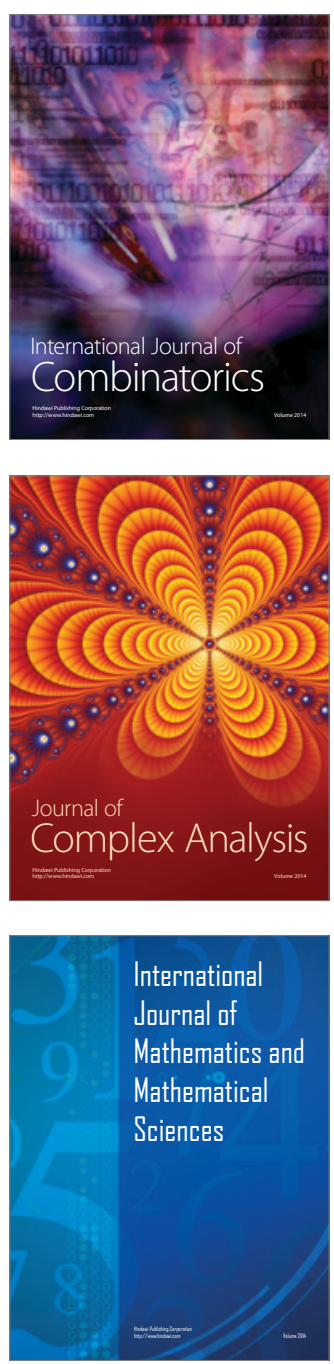
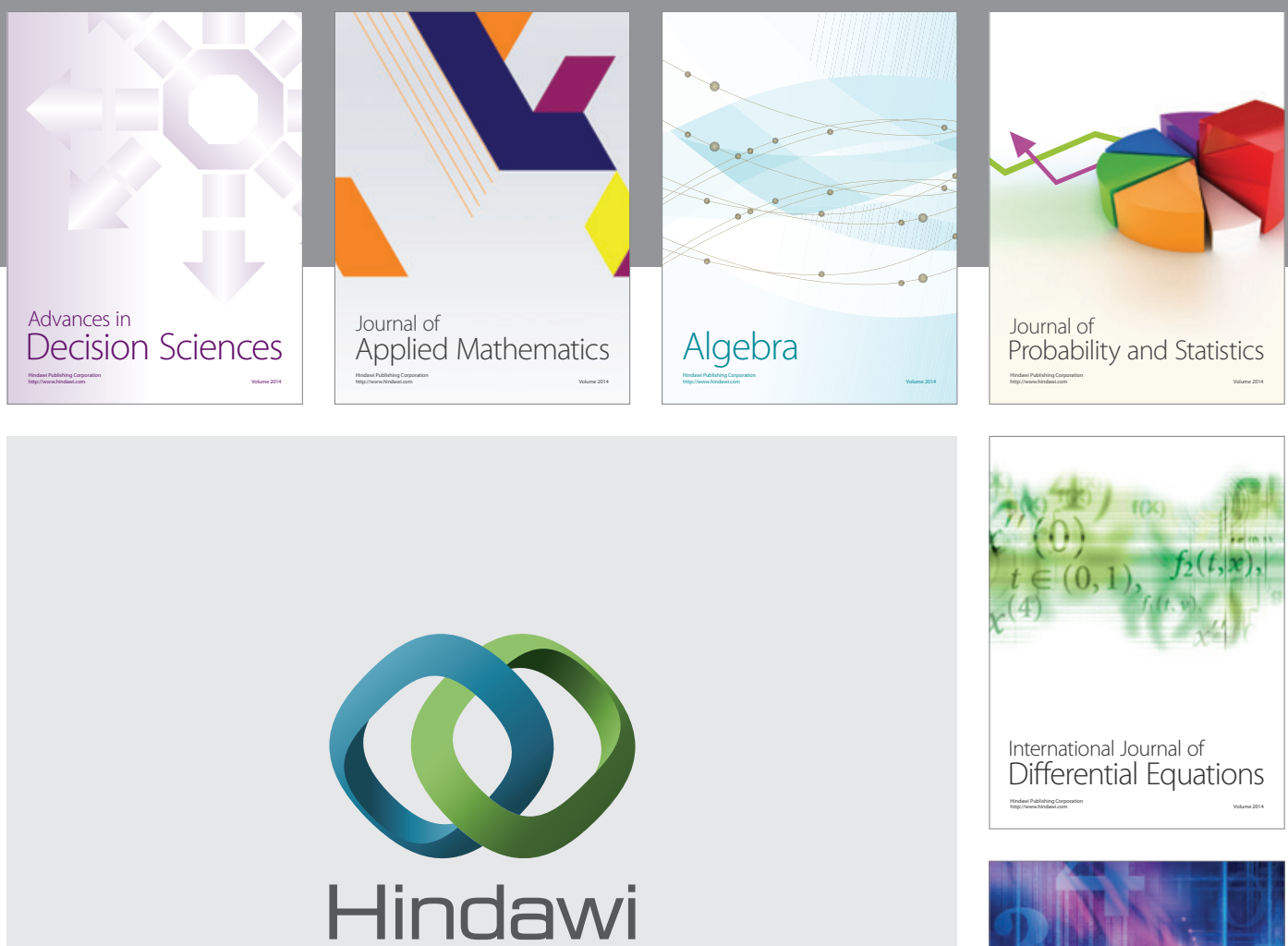

Submit your manuscripts at http://www.hindawi.com
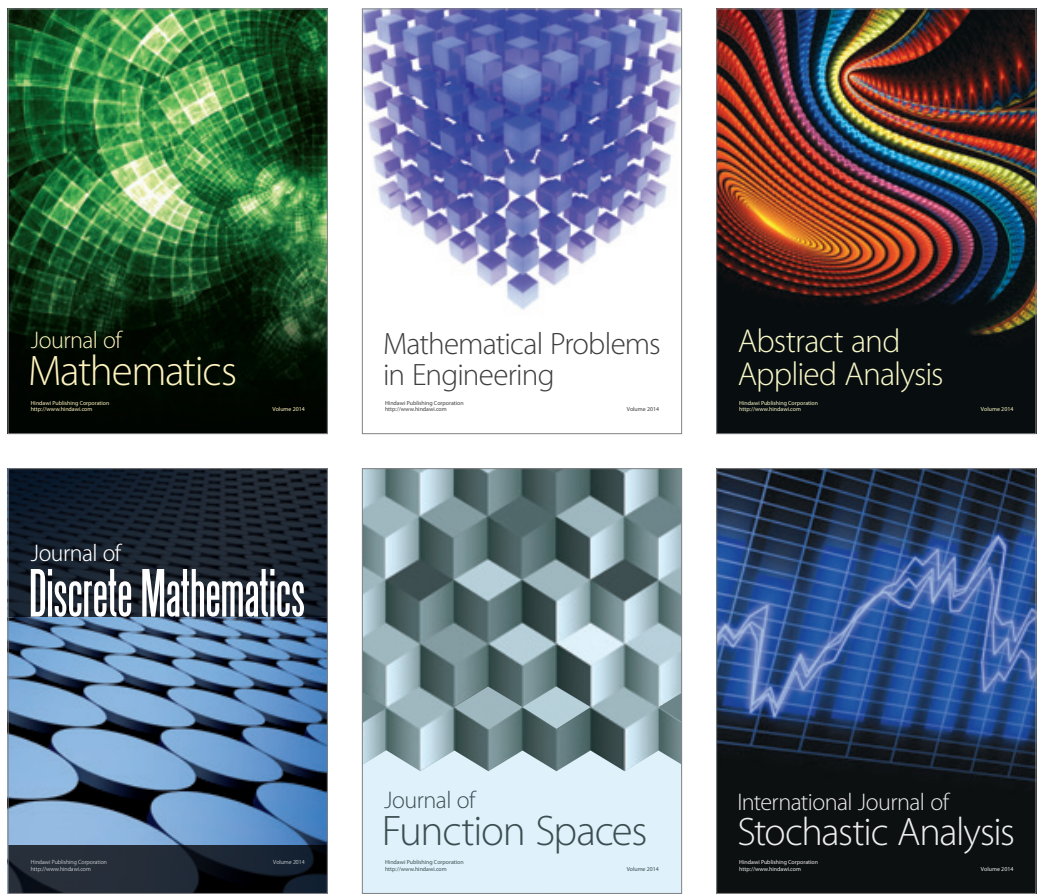

Journal of

Function Spaces

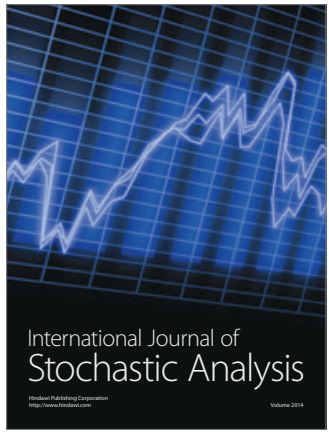

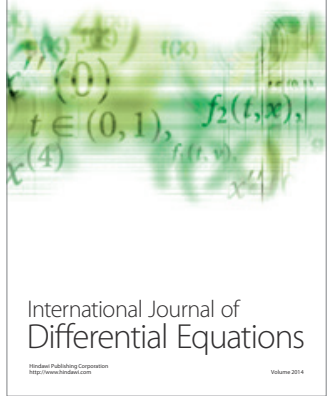
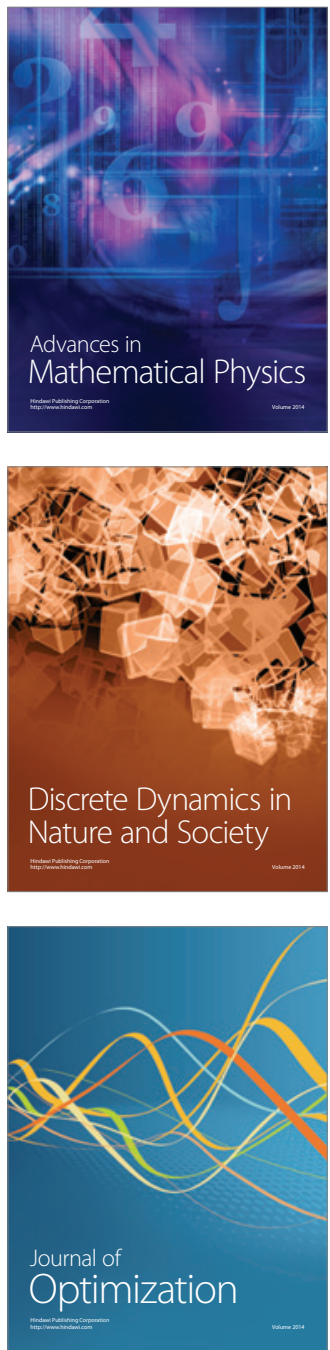